\title{
SUBSEQUENCES OF ZEROS FOR CLASSES OF HOLOMORPHIC FUNCTIONS, THEIR STABILITY, AND THE ENTROPY OF ARCWISE CONNECTEDNESS. I
}

\author{
B. N. KHABIBULLIN, F. B. KHABIBULLIN, AND L. YU. CHEREDNIKOVA
}

\begin{abstract}
For a domain $\Omega$ in the complex plane $\mathbb{C}$, let $H(\Omega)$ denote the space of functions holomorphic in $\Omega$, and let $\mathcal{P}$ be a family of functions subharmonic in $\Omega$. Denote by $H_{\mathcal{P}}(\Omega)$ the class of $f \in H(\Omega)$ satisfying $|f(z)| \leq C_{f} \exp p_{f}(z), z \in \Omega$, where $p_{f} \in \mathcal{P}$ and $C_{f}$ is a constant. The paper is aimed at conditions for a set $\Lambda \subset \Omega$ to be included in the zero set of some nonzero function in $H_{\mathcal{P}}(\Omega)$. In the first part, certain preparatory theorems are established concerning "quenching" the growth of a subharmonic function by adding to it a function of the form $\log |h|$, where $h$ is a nonzero function in $H(\Omega)$. The method is based on the balayage of measures and subharmonic functions.
\end{abstract}

\section{INTRODUCTION}

Nevanlinna's classical ultimate description of the zero sets for the algebra $H^{\infty}$ of bounded holomorphic functions in the unit disk $\mathbb{D}=\{z \in \mathbb{C}:|z|<1\}$ ( $\mathbb{C}$ is the complex plane) and similar results for the classes of Nevanlinna, Nevanlinna-Dzhrbashyan, and Hardy gave rise to a wide range of related studies for a variety of weighted algebras and spaces of holomorphic functions in $\mathbb{D}$. For the topic so broad and rich in results, we have made no attempt to achieve even a minimal coverage of the relevant bibliography. We only refer to the surveys 1 by Shvedenko, 2 by Aleksandrov, 3 by Hedenmalm, and [4] by Colwell, as well as to the treatment of spaces and algebras much similar to those studied here: the monograph $[5$ by Dzhrbashyan and Shamoyan, the final results [6. 7] by Shamoyan (a far-reaching development of Dzhrbashyan's work), the results of Horowitz [8] (on algebras of functions of moderate "exponential" or fast growth), the work of Korenblum [9], of Beller and Horowitz [10, 11, of Seip [12, 13, of Bruna and Massaneda [14, of Lueking [15] (where, in particular, stability conditions for a subsequence of zeros for Bergman-type spaces were treated), the joint monograph [16] by Hedenmalm, Korenblum, and Zhu, and the paper [17] by Blasco, Kukuryka, and Nowak (concerning spaces and algebras of functions of slow "power" growth). The Introductions and reference lists in these papers and books can give the reader an idea about the recent state of the art in the topic in question.

In the present study, we concentrate on conditions for a sequence of points in a domain $\Omega \subset \mathbb{C}$ to be a subsequence of zeros of a nontrivial function holomorphic in $\Omega$ and belonging to a certain class; these classes will be defined by growth restrictions near

2000 Mathematics Subject Classification. Primary 30C15.

Key words and phrases. Holomorphic function, algebra of functions, weighted spaces, nonuniqueness sequence.

Supported by RFBR, grant no. 06-01-00067, and by the Program of state subventions for leading scientific schools, grant NSh-10052.2006.1. 
the boundary expressed in terms of pointwise estimates via a system of subharmonic majorants.

In what follows, $\mathbb{N}, \mathbb{Z}, \mathbb{R}$, and $\mathbb{C}$ stand for the sets of natural, integer, real, and complex numbers, or their geometric interpretations.

Throughout, the positivity of a number, function, measure, etc. is understood in the nonstrict sense. Accordingly, "negativity" means nonstrict negativity. We write " $f \equiv b$ on $A$ " to indicate that $f$ is identically equal to $b$ on a set $A$, and $f \not \equiv b$ on $A$ " to indicate the opposite.

Let $\Lambda=\left\{\lambda_{k}\right\}, k=1,2, \ldots$, be a sequence of points in a domain $\Omega \subset \mathbb{C}$ without limit points in $\Omega$. We admit repetition of points $\lambda_{k}$, and also allow the sequence $\Lambda$ to be finite or empty. With every sequence $\Lambda$, we associate the counting measure $n_{\Lambda}$ on $\Omega$ as follows:

$$
n_{\Lambda}(S):=\sum_{\lambda_{k} \in S} 1, \quad S \subset \Omega,
$$

i.e., $n_{\lambda}(S)$ is the number of points $\lambda_{k}$ belonging to $S$. We deviate from the traditional interpretation of a sequence as a function and agree that $\Lambda$ coincides with another sequence $\Gamma=\left\{\gamma_{k}\right\}$ (in symbols: $\Lambda=\Gamma$ ) if the corresponding counting measures coincide: $n_{\Lambda}=n_{\Gamma}$. The inclusion $\Lambda \subset \Gamma$ means that $n_{\Lambda} \leq n_{\Gamma}$ on $\Omega$. A sequence $\Lambda$ is included in a set $S$ (in symbols: $\Lambda \subset S$ ) if the support $\operatorname{supp} n_{\Lambda}$ of $n_{\Lambda}$ is included in $S$.

We use the notation $\Lambda=\left(\lambda_{k}\right)$ when treating the sequence $\Lambda$ in the usual sense, i.e., with its enumeration.

Let $f$ be a holomorphic function in $\Omega$. If $f \not \equiv 0$, the sequence Zero $f$ of zeros of $f$ is defined to consist of points of $\Omega$ at which $f$ vanishes, each repeated the number of times equal to the multiplicity of this particular zero. If $f \equiv 0$, we agree to attribute all points of $\Omega$ to Zero $_{f}$, each repeated countably many times. A classical Weierstrass theorem says that, for every sequence $\Lambda$ in $\Omega$, there exists a function $f_{\Lambda}$ holomorphic in $\Omega$ with Zero $_{f_{\Lambda}}=\Lambda$. A sequence $\Lambda$ in $\Omega$ is said to be a subsequence of zeros for a function $f$ if $\Lambda \subset \operatorname{Zero}_{f}$.

Let $H$ be a class of functions holomorphic in $\Omega$, and $\Lambda$ a sequence of points in $\Omega$. If there exists $f \in H$ such that Zero $f=\Lambda$, we say that $\Lambda$ is a sequence of zeros for $H$. If $\Lambda$ is a subsequence of zeros for some function $f \not \equiv 0$ in $H$, we say that $\Lambda$ is a subsequence of zeros for $H$. Clearly, every sequence of zeros is also a subsequence of zeros for $H$. If $H$ is a linear space (over $\mathbb{C}$ or $\mathbb{R}$ ), a subsequence $\Lambda$ of zeros is also called a nonuniqueness sequence for $H$; accordingly, a uniqueness sequence means "not a subsequence of zeros".

We denote by $H(\Omega)$ the space (linear over $\mathbb{C}$ ) of all functions holomorphic in a domain $\Omega \subset \mathbb{C}$. We consider the following subclasses of $H(\Omega)$.

Let $p$ be a function taking values in the extended real line $[-\infty,+\infty]$. The space (linear over $\mathbb{C}$ ) of all functions $f \in H(\Omega)$ that satisfy the inequality $|f(z)| \leq C_{f} \exp p(z)$, $z \in \Omega$, where $C_{f} \geq 0$ is a constant, is denoted by $H_{p}(\Omega)$ (not to be confused with the Hardy class $\left.H^{p}(\mathbb{D})\right)$.

The symbol $S H(\Omega)$ denotes the cone of all functions subharmonic in a domain $\Omega \subset \mathbb{C}$, including the function $u \equiv-\infty$ on $\Omega$; let $S H^{+}(\Omega)$ stand for the subcone of all positive functions in $S H(\Omega)$.

Let $\mathcal{P}$ be a subset of $S H(\Omega)$ not containing the function identically equal to $-\infty$. In what follows, such a subset is called a system of weights on $\Omega$, and the functions in $\mathcal{P}$ are called weight functions, or weights. If a system $\mathcal{P}$ of weights has the property

$\left(\mathrm{H}^{\uparrow}\right)$ for every $p_{1}, p_{2} \in \mathcal{P}$ there is $p \in \mathcal{P}$ and a constant $C$ such that $\max \left\{p_{1}(z), p_{2}(z)\right\} \leq$ $p(z)+C$ for all $z \in \Omega$,

then the class $H_{\mathcal{P}}^{\uparrow}(\Omega) \stackrel{\text { def }}{=} \bigcup_{p \in \mathcal{P}} H_{p}(\Omega)$ is a linear space over $\mathbb{C}$. In particular, if $\mathcal{P}=\{p\}$ is a singleton, then condition $\left(\mathrm{H}^{\uparrow}\right)$ is fulfilled and $H_{\mathcal{P}}^{\uparrow}(\Omega)=H_{p}(\Omega)$. If $p \in S H^{+}(\Omega)$, then 
the system $\mathcal{P}=\{c p: c \in \mathbb{R}, 0 \leq c<1\}$ of weights obeys condition $\left(\mathrm{H}^{\uparrow}\right)$, and we denote $H_{\mathcal{P}}^{\uparrow}(\Omega)$ by $H_{p}^{1}(\Omega)$.

If a system $\mathcal{P}$ of weights has the property

$\left(\mathrm{A}^{\uparrow}\right)$ for every $p_{1}, p_{2} \in \mathcal{P}$ there is $p \in \mathcal{P}$ and a constant $C$ such that $p_{1}(z)+p_{2}(z) \leq$ $p(z)+C$ for all $z \in \Omega$,

then the class $H_{\mathcal{P}}^{\uparrow}(\Omega)$ is denoted by $A_{\mathcal{P}}^{\uparrow}(\Omega)$. If $\left(\mathrm{A}^{\uparrow}\right)$ and $\left(\mathrm{H}^{\uparrow}\right)$ are fulfilled simultaneously, then $A_{\mathcal{P}}^{\uparrow}(\Omega)$ is an algebra. If $p \in S H(\Omega)$ and the system $\mathcal{P}$ has the form

$$
\{c p: c \in \mathbb{R}, 0<c<+\infty\},
$$

then $\left(\mathrm{A}^{\uparrow}\right)$ is fulfilled, and we denote $A_{\mathcal{P}}^{\uparrow}(\Omega)$ by $A_{p}^{\infty}(\Omega)$. If $\mathcal{P} \subset S H^{+}(\Omega)$ and $\left(\mathrm{A}^{\uparrow}\right)$ is fulfilled, then $\left(\mathrm{H}^{\uparrow}\right)$ is also fulfilled, i.e., $A_{\mathcal{P}}^{\uparrow}(\Omega)$ is an algebra in this case. If $p \in S H^{+}(\Omega)$ and the system $\mathcal{P}$ is of the form (0.2), then both $\left(\mathrm{H}^{\uparrow}\right)$ and $\left(\mathrm{A}^{\uparrow}\right)$ are fulfilled, and $A_{p}^{\infty}(\Omega)$ is an algebra.

For the spaces $H_{\mathcal{P}}^{\uparrow}(\mathbb{D})$ and $A_{\mathcal{P}}^{\uparrow}(\mathbb{D})$, in all cases known to us where (sub)sequences of zeros have been described, the system $\mathcal{P}$ is assumed to consist of positive weights that are radial (i.e., depend only on $|z|, z \in \mathbb{D}$ ) and often are specified in quite explicit terms. Similar problems for spaces on arbitrary bounded domains have received almost no treatment previously.

There are general statements about identification of the topological dual to quite general spaces of the form $H_{\mathcal{P}}^{\uparrow}(\mathbb{D})$ and $A_{\mathcal{P}}^{\uparrow}(\mathbb{D})$, treated as projective limits of spaces of type $H_{p}(\Omega)$. These duals are realized as spaces of entire functions that are inductive limits of weighted spaces (see, e.g., [18). Mostly, this duality is reflexive. In this framework, statements about (sub)sequences of zeros for $H_{\mathcal{P}}^{\uparrow}(\mathbb{D})$ and $A_{\mathcal{P}}^{\uparrow}(\mathbb{D})$ often give certain "dual" statements about conjugate weighted spaces of entire functions (realized as inductive limits). In many questions of the theory of functions (such as completeness, interpolation, local description of ideals and submodules, spectral synthesis problems, etc.) a necessary or sufficient requirement is often that of $\Lambda$ to be a (non)uniqueness sequence for some weighted space. This motivates the treatment of general weight systems $\mathcal{P}$ rather than single weights $p$. Often, a subsequence of zeros for a class of functions need not be a sequence of zeros for this class. This feature is most probable if the weighted class is defined in terms of essentially nonradia 1 weights. This makes the study of subsequences (rather than only sequences) of zeros for weighted classes topical. Moreover, there is another general procedure (developed by the first author; see [19, 20]), which makes it possible to learn if a subsequence $\Lambda$ of zeros (of nonuniqueness) for a weighted space $H$ of holomorphic functions is also a sequence of zeros for $H$ or for other (minimal if possible) space $H^{\prime} \supset H$ of functions holomorphic in $\Omega$. This procedure converts sufficient conditions for being a subsequence of zeros to those of being a sequence of zeros.

In the present study, we give sufficient conditions for a sequence $\Lambda$ in $\Omega$ to be a subsequence of zeros for classes of the form $H_{\mathcal{P}}^{\uparrow}(\Omega)$ and $A_{\mathcal{P}}^{\uparrow}(\Omega)$. An exact criterion can hardly be expected for arbitrary domains $\Omega$ and fairly general systems $\mathcal{P}$ of weights. However, comparison of our results (see $\S 19$ in the second part) with known facts about spaces in the disk shows that, in the terms in question, our results are sharp at least for algebras.

Our main statements are new already for the algebras $A_{\mathcal{P}}^{\uparrow}(\Omega)$ with radial weights of fairly general form, and they well agree with the known descriptions of sequences of zeros for such algebras in the case of more or less specific systems $\mathcal{P}$ of weights.

\footnotetext{
${ }^{1}$ For a domain $\Omega \neq \mathbb{C}$ with nonpolar boundary, the property of a weight $p \in \mathcal{P}$ to be radial should be understood as its constancy on each level line for the Green function $g_{\Omega}$ with pole at a fixed point in $\Omega$. In general, we do not require this in the present paper.
} 
Furthermore, they cover also the case of nonradial weights $p \in \mathcal{P}$ of variable sign, and of arbitrary (including multiply connected, up to infinitely many components in the complement) domains $\Omega$. Final results (see Part II) are stated only for a bounded domain $\Omega$. Surely, the case of an unbounded $\Omega$ whose complement has nonempty interior reduces to this: it suffice to make an inversion with respect to a circle bounding a disk outside $\Omega$. However, we postpone the treatment of arbitrary unbounded domains $\Omega$ to future time. Mainly, this is related to the specific form taken by certain geometric notions in the case of an unbounded domain (the spherical, or chordal, distance arises in place of the more transparent Euclidean distance used here; see the remark at the end of $\$ 2$ ). A preliminary attempt of a unified presentation of this material both for bounded and for unbounded domains showed that (at least in the framework we have adopted) a considerable extension of the scope of investigation is needed (moreover, at the expense of geometric clarity). At the same time, potentially, the key preparatory material in $\S \S 7$ and 8 , as well as some other facts and notions, should suffice to cover the case of any unbounded domain $\Omega$.

Let $p \not \equiv-\infty$ be a subharmonic function in a domain $\Omega$. The positive measure $\nu_{p}=$ $\frac{1}{2 \pi} \Delta p$ (here $\Delta$ is the Laplace operator, and the formula is understood in the sense of distributions) is called the Riesz measure of $p$ in $\Omega$. In particular, if $f \in H(\Omega)$ and $f \not \equiv 0$, then $n_{\text {Zero }_{f}}$ is the Riesz measure for $\log |f| \in S H(\Omega)$.

Given a set $D \subset \mathbb{C}$, the symbols $\bar{D}, \partial D$, $\operatorname{diam} D$, and conv $D$ denote (respectively) the closure, boundary, diameter, and convex hull of $D$ in $\mathbb{C}$. In particular, $\overline{\mathbb{C}}$ is $\mathbb{C}$ rather than the Riemann sphere, and $\partial \mathbb{C}=\varnothing$. If $\bar{S}$ is compact in $D$ in the topology induced from $\mathbb{C}$, then $S$ is said to be precompact in $D$ (in symbols: $S \Subset D$ ).

For $z \in \mathbb{C}$ and $S, D \subset \mathbb{C}$, we denote by $\operatorname{dist}(z, D)$ and $\operatorname{dist}(S, D)$ the Euclidean distance from $z$ or $S$ to $D$. In particular, $\operatorname{dist}(z, \partial \mathbb{C})=+\infty$ and $\operatorname{dist}(S, \partial \mathbb{C})=+\infty$ because inf $\varnothing=+\infty$.

Suppose a family $\left\{S_{k}\right\}, S_{k} \Subset \Omega, k \in \mathbb{N}$, of Borel sets forms a covering of the support of a sequence $\Lambda$. It is natural to expect (see, for instance 2 [ 6 , Theorem 2.2]) that if the covering $\left\{S_{k}\right\}$ of $\Lambda$ is sufficiently "fine" and for some $p \in \mathcal{P}$ the quantity $n_{\Lambda}\left(S_{k}\right)$ is dominated by $\nu_{p}\left(S_{k}\right)$ ( $\nu_{p}$ is the Riesz measure of $p$ ), then $\Lambda$ is a (sub)sequence of zeros for $H_{\mathcal{P}}^{\uparrow}(\Omega)$. The precise meaning of the term "fine" should be in agreement with the rate of growth of the weights $p \in \mathcal{P}$ near the boundary, and also with the "gaps" between different functions in $\mathcal{P}$. The main results of the paper are explicit quantitative versions of this observation. Statements of this kind will be referred to as "statements in terms of a covering". The minimal initial requirement about gaps between functions in $\mathcal{P}$ which make our method efficient is the condition

(L) for every $p \in \mathcal{P}$, there exists $p_{1} \in \mathcal{P}$ and a constant $C$ such that

$$
p(z)+\log \left(1+\frac{1}{\operatorname{dist}(z, \partial \Omega)}\right)+\log (1+|z|) \leq p_{1}(z)+C \text { for every } z \in \Omega,
$$

where on the left the summand $\log (1+|z|)$ is redundant if $\Omega \neq \mathbb{C}$, and otherwise the summand $\log (1+1 / \operatorname{dist}(z, \partial \Omega))$ is redundant.

In a sense, the most "rigid" class of functions to which our method is applicable is the space $H_{\mathcal{P}}^{\uparrow}(\Omega)$ constructed by using the following system of weights: fix $p \in S H(\Omega)$, $p \not \equiv-\infty$, and put

$$
\mathcal{P}=\left\{p(z)+C_{1} \log \left(1+\frac{1}{\operatorname{dist}(z, \partial \Omega)}\right)+C_{2} \log (1+|z|): C_{1}, C_{2} \geq 0\right\}, \quad z \in \Omega,
$$

\footnotetext{
${ }^{2}$ It seems that Theorem 2.2 in [6] must in fact be Theorem 2.1: in [6] Remark 2.1] Theorem 2.2 is referred to as Theorem 2.1 (moreover, this theorem is at the very beginning of $\S 2$ ).
} 
where $C_{2}=0$ if $\Omega \neq \mathbb{C}$. In the sequel, we denote this space by $H_{p+\log }(\Omega)$.

In the topics studied here, even for a simply connected domain $\Omega$, reduction to the unit disk by a conformal mapping is highly conjectural because of a possible high distortion of the covering $\left\{S_{k}\right\}$ and weight functions $p \in \mathcal{P}$.

The method employed here stems from a similar study of spaces of analytic functions in 21-23. (see also a presentation of some elements of this method in the book 24, Chapter IIIC] and in the survey [25]). A general outline of the method was presented in 26] for one complex variable and in 27] for several complex variables. It differs from traditional approaches by its nonconstructive nature in the sense that no function $f \not \equiv 0$ in a weighted class and with a given subsequence of zeros is required to be constructed in an explicit form (as a product, or integral, etc.). As in [23], this approach has proved to be acceptable also in the study of stability (under "small" variations) of subsequences $\Lambda$ of zeros for weighted classes.

We note in passing that the tools employed here (i.e., measures and Jensen potentials) or, more generally, the method of balayag $\rfloor^{3}$ have been successfully used also in the opposite direction, i.e., for obtaining sufficient conditions for a sequence to be a uniqueness sequence for weighted spaces of holomorphic functions in a domain (see, e.g., [28] for spaces of entire functions; or [29, where domains in $\mathbb{C}^{n}, n>1$, are considered, but the results can easily be adapted to the one-dimensional case and remain substantial; or [30]). In certain (more or less specific) settings, necessary conditions for nonuniqueness obtained in this way may match sufficient conditions in terms of a covering found here, or may be very close to them. But this is a theme of an additional study still pending, and will not be discussed here, in particular, because of volume restrictions.

The paper is organized as follows.

In Chapter I, we study auxiliary (but not secondary) results and notions. In $\S 1$, the general balayage method is presented in a specific form. In $\S 2$, the important notion is introduced, namely, the entropy of arcwise connectedness for a subset in a domain. In $\S \S 3-5$ some aspects touched upon in $\S \S 1$ and 2 are linked to the classical notions of potential theory in the plane, such as harmonic measure, the Green function, the Harnack distance, and balayage. Finally, in $\S 6$ of Chapter I special estimates of integrals are presented (these estimates are related to Jensen measures and potentials).

Chapter II is devoted to preparatory theorems in terms of coverings for classes of type $A_{\mathcal{P}}^{\uparrow}(\Omega)$ (see Theorem 7.1 in $\S 7$ ) and $H_{\mathcal{P}}^{\uparrow}(\Omega)$ (see Theorems 8.18 .3 in $\S 8$ ). These statements cover also the case of arbitrary unbounded domains $\Omega \subset \mathbb{C}$.

The next Chapter III (in the second part of this paper, which follows) studies the properties of the entropy of arcwise connectedness, relative to the possibility of inscribing locally finite families of subsets of a bounded domain $\Omega \Subset \mathbb{C}$ into suitable families of subdomains in $\Omega(\S 9)$; to the notions of blowup and a star of a subset of $\Omega(\S 10)$; to the diameter and the segmental or convex hull of a subset ( $\$ 11)$; and to estimates for systems of positive weights $(\S 12)$.

In the last Chapter IV, we establish our main results for subsequences of zeros and sequences of nonuniqueness for weighted classes of holomorphic functions. For the algebra $A_{\mathcal{P}}^{\uparrow}(\Omega)$ with a positive system of weights, sufficient conditions for nonuniqueness are presented in $\S 13$; the case of weights of variable sign is treated in $\S 14$. Similar conditions for $H_{p}^{1}(\Omega)$ with $p \in S H^{+}(\Omega)$ and for $H_{p+\log }(\Omega)$ with $p \in S H(\Omega)$, and also for the intermediate classes $H_{p+\delta}(S)$ are studied, respectively, in $\S \S 15,16$, and 17 . In $\S 18$, we present conditions sufficient for the stability of subsequences of zeros or uniqueness sequences for weighted spaces or algebras mentioned above, under variations that are

\footnotetext{
${ }^{3}$ It can also be called the envelope method, or the method of greatest minorant (least majorant), depending on where the emphasis is.
} 
"small" in a certain sense. Finally, in $§ 19$ we illustrate the main results by the example of weighted classes of holomorphic functions in the unit disk $\mathbb{D}$ in the case of a system of radial weights, indicate the limits of applicability for these results, show connections with some known descriptions of the sequences of zeros for certain weighted algebras in $\mathbb{D}$ with radial weights, and discuss prospects for further development.

General conditions for $\Lambda$ to be a nonuniqueness sequence for type $A_{\mathcal{P}}^{\uparrow}(\mathbb{D})$ algebras were reported at an International Conference in Ufa (see [31) and at the VI Kazan Summer School-Conference in 2003 (see 32]). A complete justification of these announcements for algebras of functions in the disk can be found in 33. A stability result for nonuniqueness in the case of type $A_{\mathcal{P}}^{\uparrow}(\mathbb{D})$ algebras was discussed in 34 . A large part of the material of the present paper devoted to type $H_{\mathcal{P}}^{\uparrow}(\Omega)$ spaces was exposed in the proceedings [35] of the International School-Seminar "Geometric analysis and its applications" (Volgograd, 2004), and was announced in [36.

We illustrate the main results of the second part of the present paper concerning sequences of nonuniqueness and their stability in a simple and weak form for special cases of weighted algebras and spaces in a bounded domain $\Omega$. A function $p \in S H(\Omega)$ with Riesz measure $\nu_{p}$ will be subject to conditions of the form

(0.5) $\frac{1}{2 \pi} \int_{0}^{2 \pi} p\left(z+\varepsilon \operatorname{dist}(z, \partial \Omega) e^{i \theta}\right) d \theta+a \log \left(1+\frac{1}{\operatorname{dist}(z, \partial \Omega)}\right) \leq b p(z)+C, \quad z \in \Omega$.

Theorem 0.1 (Nonuniqueness). Let $\left\{S_{k}\right\}, k=1,2, \ldots$, be a family of mutually disjoint Borel precompact subsets of $\Omega$ such that every compact subset of $\Omega$ intersects only finitely many sets $S_{k}$. Suppose that a sequence $\Lambda$ is included in $\bigcup_{k=1}^{\infty} S_{k}$. Then:

$\left(\mathrm{U}_{1}\right)$ if $p \in S H^{+}(\Omega)$ and for $a=1$ there is $\varepsilon, 0<\varepsilon<1$, and $b, C \geq 0$ such that (0.5) is true and $\lim \sup _{k \rightarrow \infty} \frac{\operatorname{diam} S_{k}}{\operatorname{dist}\left(S_{k}, \partial \Omega\right)}<2$, $\limsup _{k \rightarrow \infty} \frac{n_{\Lambda}\left(S_{k}\right)}{\nu_{p}\left(S_{k}\right)}<+\infty$, then $\Lambda$ is a sequence of nonuniqueness for the algebra $A_{p}^{\infty}(\Omega)$;

$\left(\mathrm{U}_{2}\right)$ if for $a=1$ and for every $\varepsilon, 0<\varepsilon<1$, there exist $b, C \geq 0$, such that (0.5) is true and $\lim \sup _{k \rightarrow \infty} \frac{\operatorname{diam} S_{k}}{\operatorname{dist}\left(S_{k}, \partial \Omega\right)}<1$, $\limsup _{k \rightarrow \infty} \frac{n_{\Lambda}\left(S_{k}\right)}{\nu_{p}\left(S_{k}\right)}<+\infty$, then $\Lambda$ is a subsequence of zeros for $A_{p}^{\infty}(\Omega)$;

$\left(\mathrm{U}_{3}\right)$ if $p \in S H^{+}(\Omega)$ and for $a=1$ and every $b>1$ there exists $\varepsilon, 0<\varepsilon<1$, and $C \geq 0$ such that (0.5) is true and $\lim _{k \rightarrow \infty} \frac{\operatorname{diam} S_{k}}{\operatorname{dist}\left(S_{k}, \partial \Omega\right)}=0$, $\lim _{\sup _{k \rightarrow \infty}} \frac{n_{\Lambda}\left(S_{k}\right)}{\nu_{p}\left(S_{k}\right)}<1$, then $\Lambda$ is a sequence of nonuniqueness for $H_{p}^{1}(\Omega)$;

$\left(\mathrm{U}_{4}\right)$ if for $b=1$ there exists $\varepsilon, 0<\varepsilon<1, C \geq 0$, and $a<0$ such that (0.5) is true and $\sum_{k=1}^{\infty} \frac{\operatorname{diam} S_{k}}{\operatorname{dist}\left(S_{k}, \partial \Omega\right)} \nu_{p}\left(S_{k}\right)<+\infty$ as well as $n_{\Lambda}\left(S_{k}\right) \leq \nu_{p}\left(S_{k}\right)$ for all sufficiently large $k$, then $\Lambda$ is a sequence of nonuniqueness for $H_{p+\log }(\Omega)$.

Statements $\left(\mathrm{U}_{1}\right)-\left(\mathrm{U}_{4}\right)$ are consequences of Theorems 13.1, 14.1, 15.1, and 16.1, respectively (see the remarks in $\S \S 13-16$ ).

In stability theorems, a particular enumeration of a sequence of points in a domain will be important.

Theorem 0.2 (Stability). Suppose $\Lambda=\left(\lambda_{k}\right)$ and $\Gamma=\left(\gamma_{k}\right), k=1,2, \ldots$, are two sequences in $\Omega$ without limit points in $\Omega$. Then:

$\left(\mathrm{S}_{1}\right)$ if $\lim \sup _{k \rightarrow \infty} \frac{\left|\lambda_{k}-\gamma_{k}\right|}{\min \left\{\operatorname{dist}\left(\lambda_{k}, \partial \Omega\right), \operatorname{dist}\left(\gamma_{k}, \partial \Omega\right)\right\}}<2$ and $p$ is as in $\left(\mathrm{U}_{1}\right)$, then $\Lambda$ and $\Gamma$ can be uniqueness sequences for the algebra $A_{p}^{\infty}(\Omega)$ only simultaneously;

$\left(\mathrm{S}_{2}\right)$ if $\lim \sup _{k \rightarrow \infty} \frac{\left|\lambda_{k}-\gamma_{k}\right|}{\min \left\{\operatorname{dist}\left(\lambda_{k}, \partial \Omega\right), \operatorname{dist}\left(\gamma_{k}, \partial \Omega\right)\right\}}<1$ and $p$ is as in $\left(\mathrm{U}_{2}\right)$, then $\Lambda$ and $\Gamma$ can be subsequences of zeros for $A_{p}^{\infty}(\Omega)$ only simultaneously; 
$\left(\mathrm{S}_{3}\right)$ if $\lim _{k \rightarrow \infty} \frac{\left|\lambda_{k}-\gamma_{k}\right|}{\min \left\{\operatorname{dist}\left(\lambda_{k}, \partial \Omega\right), \operatorname{dist}\left(\gamma_{k}, \partial \Omega\right)\right\}}=0$ and $p$ is as in $\left(\mathrm{U}_{3}\right)$, then $\Lambda$ and $\Gamma$ can be uniqueness sequences for $H_{p}^{1}(\Omega)$ only simultaneously;

$\left(\mathrm{S}_{4}\right)$ if $\sum_{k=1}^{\infty} \frac{\left|\lambda_{k}-\gamma_{k}\right|}{\min \left\{\operatorname{dist}\left(\lambda_{k}, \partial \Omega\right), \operatorname{dist}\left(\gamma_{k}, \partial \Omega\right)\right\}}<+\infty$ and $p$ is as in $\left(\mathrm{U}_{4}\right)$, then $\Lambda$ and $\Gamma$ can be uniqueness sequences for $H_{p+\log }(\Omega)$ only simultaneously.

Statements $\left(\mathrm{S}_{1}\right)-\left(\mathrm{S}_{4}\right)$ are obvious consequences of Theorems 18.1-18.4 in second part of the paper.

For domains $\Omega$ of a special type, the assumptions of the nonuniqueness or stability theorems can be considerably relaxed. For instance, if $\Omega$ is convex, we may replace "2" by " $+\infty$ " on the right of the inequalities in $\left(\mathrm{U}_{1}\right)$ and $\left(\mathrm{S}_{1}\right)$.

On the left in (0.5), the average over the circle $\partial D(z, \varepsilon \operatorname{dist}(z, \partial \Omega))$ occurs. Preparatory theorems of Chapter II are sufficiently general to allow for a replacement of such averages by certain averages with a more complicated dependence on $\operatorname{dist}(\cdot, \partial \Omega)$. An additional comment to the point will be given at the end of Subsection 1 in $\S 19$, after Corollary 19.2.

The symbol $\square$ marks the end of a proof. However, we use to mark the end of the proof of an intermediate statement (say, a lemma) inside another proof. A number over a relation sign ( such as $=,>, \subset$, etc.) refers to the corresponding formula or statement used to justify this relation.

\section{Chapter I. Auxiliary notions And Results}

\section{§1. Jensen measures And potentials, And "Quenching" the Growth OF A SUBHARMONIC FUNCTION}

The results of this section constitute the first step in our balayage method. They were proved (with some imperfections) in the paper [37, which is not easily available. Here we present the complete proofs, to make the exposition self-contained and to eliminate these imperfections.

Throughout, $\Omega$ is a domain in $\mathbb{C}$. There is no loss of generality in assuming that $0 \in \Omega$. As usual, $C(\Omega)$ stands for the space of continuous functions on $\Omega$. The set of all functions on $\Omega$ that are locally integrable with respect to the Lebesgue measure $m$ and take values in $[-\infty,+\infty]$ is denoted by $L_{\mathrm{loc}}^{1}(\Omega)$.

The open disk of radius $t>0$ and centered at $z \in \mathbb{C}$ is denoted by $D(z, t)$. Furthermore, $D(t) \stackrel{\text { def }}{=} D(0, t), t>0$.

The notation $\mathcal{M}^{+}(\Omega)$ is reserved for the class of all positive Borel measures $\mu$ on $\Omega$; supp $\mu$ is the support of $\mu ; \mathcal{M}_{\mathrm{ac}}^{+}(\Omega)$ is the subclass of $\mathcal{M}^{+}(\Omega)$ consisting of all measures absolutely continuous with respect to Lebesgue measure.

As in the Introduction, for a function $u \in S H(\Omega), u \neq \equiv-\infty$, we denote by $\nu_{u}$ its Riesz measure; vice versa, for $\nu \in \mathcal{M}^{+}(\Omega)$, we denote by $u_{\nu}$ a subharmonic function whose Riesz measure is $\nu\left(u_{\nu}\right.$ always exists and is unique up to a harmonic summand; see [38]).

In order to prove the main result of this chapter (Theorem 1.1), we need some notions and facts from [21, 22, 26], 39]-41], and their extensions. We state them here only for the cone $S H(\Omega)$.

Definition 1.1. A measure $\mu \in \mathcal{M}^{+}(\Omega)$ with a compact support included in $\Omega$ is called a Jensen measure for $\Omega$ (at the point $0 \in \Omega$ ) if

$$
u(0) \leq \int_{\Omega} u d \mu
$$

for every function $u$ subharmonic in $\Omega$. The class of all Jensen measures is denoted by $J_{0}(\Omega)$. 
Particular cases of Jensen measures are the restriction $m^{(r)}$ of the Lebesgue measure $m$ to the disk $D(r) \Subset \Omega$ normalized by the condition $m^{(r)}(D(r))=1$, and also the arc length $s^{(r)}$ on the circle $\partial D(r)$ normalized by $s^{(r)}(\partial D(r))=1$. A larger (though not exhaustive) class of Jensen measures at 0 is given by the harmonic measures $\omega_{D}(0, \cdot)$ for subdomains $D \Subset \Omega, 0 \in D$. Clearly, every Jensen measure is of total mass 1 , and if $0 \in \Omega \subset \Omega^{\prime}$, where $\Omega^{\prime}$ is a domain in $\mathbb{C}$, then $J_{0}(\Omega) \subset J_{0}\left(\Omega^{\prime}\right)$.

A Jensen measure at 0 for $\Omega$ is a particular case of balayage. Specifically, it is a balayage of the Dirac easure $\delta_{0}$ (the unit mass at 0 ) with respect to the cone $S H(\Omega)$. Taking this into account, we can formulate a special case 4 of [27, Theorem 7.1], to be used in what follows.

Theorem 1.1. Suppose $\Omega$ is a domain in $\mathbb{C}, 0 \in \Omega$, and $F \in L_{\text {loc }}^{1}(\Omega)$. If

$$
-\infty<\inf \left\{\int_{\Omega} F d \mu: \mu \in J_{0}(\Omega) \cap \mathcal{M}_{\mathrm{ac}}^{+}(\Omega)\right\},
$$

then for every function $r \in C(\Omega)$ with

$$
0<r(z)<\operatorname{dist}(z, \partial \Omega), \quad z \in \Omega,
$$

there exists a function $v \in S H(\Omega)$ satisfying

$$
v(z) \leq F^{(r)}(z) \stackrel{\text { def }}{=} \int_{D(r(z))} F(z+\zeta) d m^{(r(z))}(\zeta), \quad z \in \Omega,
$$

and harmonic in a neighborhood of zero.

In [27, Theorem 7.1] it was proved only that it is possible to choose $v \not \equiv-\infty$. It is easy to adjust $v$ in such a way that it becomes harmonic near zero and still satisfies (1.4). For this, we observe that the function $F^{(r)}$ given by (1.4) is continuous on $\Omega$ (because $r$ is continuous and $F$ is locally integrable). The required modification of $v$ is done as follows: we extend it to be harmonic inside a sufficiently small disk centered at zero (in accordance with the definition (4.3) below, this is the balayage of $v$ from this disk), and then subtract a large positive constant from the resulting function.

Definition 1.2. Let $\mu$ be a Jensen measure for $\Omega$. The function

$$
V_{\mu}(\zeta)=\int_{\mathbb{C}} \log |z-\zeta| d \mu(z)-\log |\zeta|
$$

is called the potential of $\mu$.

The following generalization of the Poisson-Jensen formula was proved in 21, Proposition 1.4], [26, Proposition 3.3], and [27, Lemma 8.1] for a simply connected domain, and in [42, Proposition 1.2] for an arbitrary domain.

Proposition 1.1. Suppose that $0 \in \Omega$ and $\mu \in J_{0}(\Omega)$. For every subharmonic function $u$ in $\Omega$ we have

$$
\int_{\Omega} u d \mu=\int_{\Omega} V_{\mu} d \lambda_{u}+u(0),
$$

provided $u(0) \neq-\infty$. Here $\lambda_{u}$ is the Riesz measure for $u$ and $V_{\mu}$ is the potential of $\mu$.

Definition 1.3. A function $V$ subharmonic in $\Omega \backslash\{0\}$ is called 5 a Jensen potential for $\Omega$ (with pole at 0 ) if the following three conditions are satisfied:

(1) there is a compact set $K \subset \Omega$ such that $V(z) \equiv 0$ for $z \in \Omega \backslash K$;

\footnotetext{
${ }^{4}$ In [27, Theorem 7.1], $\mathbb{R}^{2}$ must be identified with $\mathbb{C}, G$ must be redenoted by $\Omega$, and $S H(\Omega)$ and $\delta_{0}$ must be taken for the cone $H$ and the measure $\nu$.

${ }^{5}$ Before, we called them "Jensen's functions" (by analogy with Green's function).
} 
(2) $V(z) \leq-\log |z|+O(1)$ as $z \rightarrow 0$;

(3) $V(\zeta) \geq 0$ for $\zeta \in \mathbb{C} \backslash\{0\}$.

The class of all Jensen potentials will be denoted by $P J_{0}(\Omega)$.

In particular, every positive Green function $g_{D}(0, z), z \in \mathbb{C}$, for the domain $D$ and with pole at 0 (see 38 and $\S 4$ below) belongs to $P J_{0}(\Omega)$. Nontraditional examples of Jensen potentials different from Green's functions can be found, e.g., in 21 .

The mapping $\mu \mapsto V_{\mu}$ is a bijection of $J_{0}(\Omega)$ onto the class $P J_{0}(\Omega)$. Moreover, the Riesz measure (in $\mathbb{C} \backslash\{0\}$ ) of $V_{\mu}$ is the restriction of $\mu$ to $\mathbb{C} \backslash\{0\}$ (see [21, Proposition 4.1] and [27, Lemma 8.3] for simply connected domains, and [22, Duality theorem] for arbitrary domains).

The general Theorem 1.2 presented below is the basis of our study of nonuniqueness sequences. It is a variation of [21, Main Theorem], 26. Theorem 1.3], and 22, Theorem 1] 6 and in terms of Jensen measures, it gives sufficient conditions for the possibility to "quench" the growth of a subharmonic function $7 u$ on $\Omega$ by adding to it a function of the form $\log |h|$, where $h \in H(\Omega), h \neq \equiv 0$. A certain refinement of these statements in the one-dimensional case is mainly due to the uniform estimate of the solution to the $\bar{\partial}$-problem found by Epifanov [43. An important difference from the above-mentioned statement is that averages of $M$ over some disks or circles (rather than the least upper bound over disks of variable radius) occur on the right in (1.8). This may become a dramatic improvement if $M$ is harmonic on some pieces of $\Omega$.

Theorem 1.2. Let $\lambda_{u}$ be the Riesz measure of a function $u \in S H(\Omega), u(0) \neq-\infty$, and let $M \in L_{\mathrm{loc}}^{1}(\Omega)$. If for some constant $C$ we have

$$
\int_{\Omega} V_{\mu} d \lambda_{u} \leq \int_{\Omega} M d \mu+C
$$

for all absolutely continuous Jensen measures $\mu$ for $\Omega$ (where $V_{\mu}$ is the potential of $\mu$ ), then for every function $r \in C(\Omega)$ satisfying (1.3) there exists a function $h$ holomorphic in $\Omega$ with $h(0) \neq 0$ and such that

$$
\begin{aligned}
u(z)+\log |h(z)| \leq & \inf _{0<t<\operatorname{dist}(z, \partial \Omega)}\left(\frac{1}{2 \pi} \int_{0}^{2 \pi} M^{(r)}\left(z+t e^{i \theta}\right) d \theta+\log (1+1 / t)\right) \\
& +9 \log (1+|z|), \quad z \in \Omega .
\end{aligned}
$$

Proof. Since $u$ is subharmonic, it follows that $u \in L_{\text {loc }}^{1}(\Omega)$ (see [38]). Then $F=M-u$ is also in $L_{\text {loc }}^{1}(\Omega)$. By the general Jensen-Poisson formula (1.6), relation (1.7) can be rewritten in the form (1.2). By Theorem 1.1, there exists a function $v \in S H(\Omega)$ that is harmonic near zero and satisfies (1.4). For $F=M-u$, (1.4) yields

$$
v(z) \leq F^{(r)}(z)=M^{(r)}(z)-u^{(r)}(z), \quad z \in \Omega .
$$

Since the subharmonic function $u$ does not exceed its average over circles, (1.9) implies

$$
u(z)+v(z) \leq M^{(r)}(z), \quad z \in \Omega .
$$

Our next task is to find a holomorphic function $h \not \equiv 0$ in $\Omega$ that can replace $v$ in (1.10) at the expense of making $M$ slightly bigger.

\footnotetext{
${ }^{6}$ Unfortunately, in the theorem on the least majorant in 22 (which plays a key role in the proof of [22. Theorem 1]), the last formula was given with a regrettable typo, and then additionally distorted in the English translation. It should read $\nu\left(\mathfrak{M}_{X} F\right)=\sup _{\nu \prec \mu} \mu(F)$.

${ }^{7}$ Starting with Chapter IV, in statements about nonuniqueness sequences $\Lambda$ we take $u=\log \left|f_{\Lambda}\right|$, where $f_{\Lambda} \in H(\Omega)$ is an arbitrary function with $\operatorname{Zero}_{f_{\Lambda}}=\Lambda$; we shall "quench" the growth of $\left|f_{\Lambda}\right|$ by a multiplier $h \in H(\Omega)$, i.e., $\Lambda \subset \operatorname{Zero}_{f_{\Lambda}} h$.
} 
Lemma 1.1. Suppose that for some $\beta>0$ a function $v \in S H(\Omega)$ satisfies

$$
\inf _{z \in D(2 \beta)} v(z)>-\infty, \quad D(2 \beta) \Subset \Omega .
$$

Then there exists $h \in H(\Omega)$ such that $h(0) \neq 0$ and for every $z \in \Omega$ and every $t$ with $0<t<\operatorname{dist}(z, \partial \Omega)$ we have

$$
\log |h(z)| \leq \frac{1}{2 \pi} \int_{0}^{2 \pi} v\left(z+t e^{i \theta}\right) d \theta+\log \left(1+\frac{1}{t}\right)+9 \log (1+|z|) .
$$

Proof of Lemma 1.1. Consider the subharmonic function

$$
\varphi(z)=v(z)+2 \log |z|, \quad z \in \Omega .
$$

Let $\chi$ be an infinitely differentiable function that vanishes off the disk $D(\beta) \Subset \Omega$ and is equal to a nonzero constant in the vicinity of the closed disk $\overline{D(\beta / 2)}$. We put

$$
g=\frac{\partial \chi}{\partial \bar{z}}, \quad z \in \Omega
$$

By the choice of $\chi, g$ is identically zero in the vicinity of $\overline{D(\beta / 2)}$ and in the vicinity of the complement $\mathbb{C} \backslash D(2 \beta)$. By (1.11), we can ensure the inequality $|g(z)| \leq e^{\varphi(z)}, z \in \Omega$ (it suffices to multiply $\chi$ by a small positive constant). In view of Epifanov's uniform version of the Hörmander theorem (see [43, Theorem 1]), there exists a weak solution $f$ of the equation

$$
\frac{\partial f}{\partial \bar{z}}=g, \quad f \in C(\Omega)
$$

satisfying

$$
|f(z)| \leq 24(1+|z|)^{4} \cdot \inf _{0<t<\operatorname{dist}(z, \partial \Omega)}\left(\frac{(1+t)^{4}}{t} \exp \left(\frac{1}{2 \pi} \int_{0}^{2 \pi} \varphi\left(z+t e^{i \theta}\right) d \theta\right)\right) .
$$

Since $g \equiv 0$ in $D(\beta / 2)$, the function $f$ is holomorphic in $D(\beta / 2)$. Next, for $z=0$ and $t>0$ sufficiently small, formulas (1.16) and (1.13) imply

$$
\begin{aligned}
|f(0)| & \leq 24 \cdot \frac{(1+t)^{4}}{t} \exp \left(\frac{1}{2 \pi} \int_{0}^{2 \pi} v\left(t e^{i \theta}\right) d \theta+2 \log t\right) \\
& =24(1+t)^{4} t \exp \left(\frac{1}{2 \pi} \int_{0}^{2 \pi} v\left(t e^{i \theta}\right) d \theta\right) .
\end{aligned}
$$

Letting $t \rightarrow 0$, we obtain $f(0)=0$.

Thus, we have found a continuous function $H$,

$$
H=\chi-f, \quad H(0)=\chi(0)-f(0)=\chi(0) \neq 0,
$$

such that (see (1.14), (1.15)) $\frac{\partial H}{\partial \bar{z}}=\frac{\partial \chi}{\partial \bar{z}}-\frac{\partial f}{\partial \bar{z}}=g-\frac{\partial f}{\partial \bar{z}}=0$ everywhere on $\Omega$. Consequently, $H \not \equiv 0$ is holomorphic in $\Omega$. Next, (1.17), (1.16), and (1.13) imply that $|H(z)|$ is bounded from above by the quantity

$$
24(1+|z|)^{4} \cdot \inf _{0<t<\operatorname{dist}(z, \partial \Omega)}\left(\frac{(1+t)^{4}}{t} \exp \left(\frac{1}{2 \pi} \int_{0}^{2 \pi} v\left(z+t e^{i \theta}\right) d \theta+2 \log (|z|+t)\right)\right)
$$

for every $z \in \Omega \backslash D(\beta)$. For $t<1+|z|$, this implies

$$
\begin{aligned}
& \log |H(z)| \leq \log 24+4 \log (1+|z|)+3 \log (2+|z|)+\log \left(1+\frac{1}{t}\right) \\
&+ 2 \log (1+2|z|)+\frac{1}{2 \pi} \int_{0}^{2 \pi} v\left(z+t e^{i \theta}\right) d \theta \\
& z \in \Omega \backslash D(\beta), \quad 0<t<\min \{\operatorname{dist}(z, \partial \Omega), 1+|z|\} .
\end{aligned}
$$


By (1.11), the right-hand side of (1.18) is bounded from below by a finite constant everywhere on $D(\beta)$. Thus, if $a>0$ is sufficiently small, we have the following estimate for the holomorphic function $a H$ under the condition that $t<1+|z|$ :

$$
\log |a H(z)| \leq 9 \log (1+|z|)+\log \left(1+\frac{1}{t}\right)+\frac{1}{2 \pi} \int_{0}^{2 \pi} v\left(z+t e^{i \theta}\right) d \theta, \quad z \in \Omega .
$$

Since the averages over circles of the subharmonic function $v$ increase monotonically, the condition $t<1+|z|$ can easily be lifted: it suffices to replace $a H$ by $h=a H / 2$ on the left. Then $h$ is a required function because it is holomorphic in $\Omega$ and $h(0) \neq 0$ by (1.17).

We finish the proof of Theorem 1.2. Averaging the two sides of (1.10) over circles centered at $z \in \Omega$ and of radii $t<\operatorname{dist}(z, \partial \Omega)$, we obtain

$$
\begin{aligned}
u(z)+\frac{1}{2 \pi} \int_{0}^{2 \pi} v\left(z+t e^{i \theta}\right) d \theta & \leq \frac{1}{2 \pi} \int_{0}^{2 \pi} u\left(z+t e^{i \theta}\right) d \theta+\frac{1}{2 \pi} \int_{0}^{2 \pi} v\left(z+t e^{i \theta}\right) d \theta \\
& \leq \frac{1}{2 \pi} \int_{0}^{2 \pi} M^{(r)}\left(z+t e^{i \theta}\right) d \theta .
\end{aligned}
$$

The function $v$ is harmonic near zero, and hence satisfies (1.11); by Lemma1.1, it satisfies (1.12), to which we add (1.20) and arrive at (1.8).

\section{§2. ENTROPy OF ARCWISE CONNECTEDNESS}

Let $S$ be a nonempty subset of a domain $D \subset \mathbb{C}$. For brevity, we redenote the distance to the boundary of $D$ as follows:

(2.15)

(2.1b)

$$
\begin{aligned}
& d_{D}(z) \stackrel{\text { def }}{=} \operatorname{dist}(z, \partial D), \quad z \in D, \\
& d_{D}(S) \stackrel{\text { def }}{=} \inf _{z \in S} d_{D}(z) \stackrel{\text { 2.1.t. }}{=} \operatorname{dist}(S, \partial D), \\
& b_{D}(S) \stackrel{\text { def }}{=} \sup _{z \in S} d_{D}(z) \stackrel{\text { 2.17] }}{=} \sup _{z \in S} \operatorname{dist}(z, \partial D) .
\end{aligned}
$$

Next, for $S \subset \mathbb{C}$ and $\varepsilon>0$ we put

$$
S^{\varepsilon} \stackrel{\text { def }}{=} \bigcup\{D(z, \varepsilon): z \in S\} ;
$$

this set will be called 8 the $\varepsilon$-blowup of $S$ (see (5.6) for a slight development of this notion). Clearly, $\bar{S}^{\varepsilon}=S^{\varepsilon}$. If $S$ (or merely $\bar{S}$ ) is connected, then $S^{\varepsilon}$ is a domain.

For references, we collect some elementary properties of the function $d_{D}$ in the next statement.

Proposition 2.1. If $D \subset \mathbb{C}$ is a bounded domain, then the function $d_{D}$ is positive and continuous, and has the following properties:

(d1) $|z-w| \geq d_{D}(z)-d_{D}(w)$ for every $z, w \in D$;

(d2) if $S \subset S^{\prime} \subset D \subset \Omega$ and $\Omega$ is a domain, then $d_{D}(S) \geq d_{D}\left(S^{\prime}\right), d_{D}(S) \leq d_{\Omega}(S)$;

$$
z, w \in D, \quad d_{D}(z) \geq d>0 \quad \text { and }|z-w| \leq d / 2,
$$

then the segment $[z, w]$ joining $z$ and $w$ lies in $D$ and $d_{D}([z, w]) \geq d / 2$;

(d4) if $d_{D}(S) \geq \varepsilon>0$ and $S \subset D$, then $S^{\varepsilon} \subset D$ and $d_{D}\left(S^{\varepsilon}\right) \geq d_{D}(S)-\varepsilon$;

(d5) if a set $S \Subset \mathbb{C}$ (or its closure) is connected, then $d_{S^{\varepsilon}}(S) \geq \varepsilon$;

\footnotetext{
8 Other authors also use the terms " $\varepsilon$-extension of $S$ " or "the set parallel to $S$ at the distance $\varepsilon$ ".
} 
(d6) the functions $\log 1 / d_{D}$ and $\log \left(1+1 / d_{D}\right)$ are subharmonic in $D$; if $D$ is convex, then $d_{D}$ is concave and $\log 1 / d_{D}$ is convex in $D$.

Proof. By (2.1z), the inequality $\operatorname{dist}(z, D) \leq|z-w|+\operatorname{dist}(w, D)$ means that $d_{D}(z) \leq$ $|z-w|+d_{D}(w)$, which is (d1). Property (d2) is an easy consequence of the definition (2.15).

If (2.3) is fulfilled, then, by (2.1E), we have $\operatorname{dist}(z, \partial D)=d_{D}(z) \geq d$, i.e., the closed disk $\overline{D(z, d / 2)}$ includes $[z, w]$ and is included in $D$. Now $(\mathrm{d} 2)$ implies $\operatorname{dist}([z, w], \partial D) \geq$ $\operatorname{dist}(D(z, d / 2), \partial D) \geq d / 2$. The definition (2.1E) yields the desired inequality $d_{D}([z, w]) \geq$ $d / 2$.

By (2.1B), properties (d4) and (d5) are consequences of the obvious inequalities $\operatorname{dist}\left(S^{\varepsilon}, \partial D\right) \geq \operatorname{dist}(S, \partial D)-\varepsilon$ and $\operatorname{dist}\left(S, \partial S^{\varepsilon}\right) \geq \varepsilon$.

For the proof of (d6), we refer the reader to [44, Theorems 2.1.24, 3.2.30 and Exercise 3.2.6].

Definition 2.1. The entropy of arcwise connectedness of a nonempty subset $S$ in a bounded domain $D \subset \mathbb{C}$ is defined to be the quantity

$$
\begin{gathered}
\ell(S ; D) \stackrel{\text { def }}{=} \sup _{z, w \in S} \inf _{l(z, w) \subset D} \frac{|l(z, w)|}{\operatorname{dist}(l(z, w), \partial D)} \\
\stackrel{\text { 2.11 }}{=} \sup _{z, w \in S} \inf _{l(z, w) \subset D} \frac{|l(z, w)|}{d_{D}(l(z, w))},
\end{gathered}
$$

where the infimum is taken over all rectifiable (not necessarily Jordan) $\operatorname{arc} 9 l(z, w) \subset D$ that join $z$ with $w$, and $|l(z, w)|$ denotes the Euclidean length of such an arc.

A precise analogy with Kolmogorov's entropy of a set in a metric space would have required taking $\log _{2} \ell(S ; D)$ for the entropy of arcwise connectedness, but in our case it is more convenient to do without logarithms. The following statement serves as a motivation.

Proposition 2.2. If $\ell(S ; D)<l<+\infty$, then for every pair of points $z, w \in S$ there is a finite sequence of disks $D\left(\zeta_{k}, d\right), k=0, \ldots, n+1$, included in $D$ and such that the following two conditions are fulfilled simultaneously:

(D1) $z=\zeta_{0}, w=\zeta_{n+1}$, and $\left|\zeta_{k-1}-\zeta_{k}\right| \leq d / 2$ for $k=1, \ldots, n+1$;

(D2) $n \leq 2 l$.

Proof. Let $z, w \in S$. By (2.4), there exists a rectifiable arc $l(z, w) \subset D$ such that

$$
\frac{|l(z, w)|}{d} \leq l, \quad d=d_{D}(l(z, w)) \stackrel{(2.1 B)}{=} \operatorname{dist}(l(z, w), \partial D) .
$$

Moving along $l$ from $z$ to $w$, we split it by consecutive points

$$
\zeta_{0}=z, \zeta_{1}, \ldots, \zeta_{n+1}=w, \quad \zeta_{k} \in l(z, w) \subset D,
$$

into subarcs $l\left(\zeta_{k-1}, \zeta_{k}\right)$ with lengths satisfying

$$
\left|l\left(\zeta_{k-1}, \zeta_{k}\right)\right|=\frac{d}{2}, k=1,2, \ldots, n ; \quad\left|l\left(\zeta_{n}, \zeta_{n+1}\right)\right| \leq \frac{d}{2} .
$$

By (2.6) and the choice of $d$ in (2.5), every disk $D\left(\zeta_{k}, d\right), k=0,1, \ldots, n+1$, is included in $D$; furthermore, (2.6) and (2.7) imply (D1). Moreover, (2.7) shows that $n d / 2=$ $\left|\bigcup_{k=1}^{n} l\left(\zeta_{k-1}, \zeta_{k}\right)\right| \leq|l(z, w)|$, and by (2.5) we obtain $n \leq 2 \frac{|l(z, w)|}{d} \leq 2 l$, which yields (D2).

\footnotetext{
${ }^{9}$ We hope that in what follows the context will enable the reader to determine in each particular case whether an arc is treated as a continuous map or as a set.
} 
In the following statement, we collect elementary consequences of Definition 2.1.

Proposition 2.3. Suppose $D$ is a bounded domain in $\mathbb{C}$ and $S \subset D$. Then:

(11) $\ell(S ; D)=\ell(\bar{S} \cap D ; D)$;

(12) if $S \subset S^{\prime} \subset D$, then $\ell(S ; D) \leq \ell\left(S^{\prime} ; D\right)$;

(13) if $\Omega$ is a bounded domain in $\mathbb{C}$ and $D \subset \Omega$, then $\ell(S ; D) \geq \ell(S ; \Omega)$;

(14) $S \Subset D$ if and only if $\ell(S ; D)<+\infty$.

Proof. Properties (11)-(13) are obvious consequences of (2.4), and only (14) needs a proof.

Let $S \Subset D$. Then there is $\varepsilon, 0<\varepsilon \leq 1$, such that

$$
\operatorname{dist}(S, \partial D) \geq 2 \varepsilon \text {. }
$$

Take a finite $\varepsilon$-net $\left\{z_{1}, \ldots, z_{N}\right\}$ for $S$ in $D$. For every pair $z_{k}, z_{m}$, we choose a rectifiable arc (say, a polygonal line with finitely many links) $l_{k m} \Subset D$ that joins $z_{k}$ and $z_{m}$. These arcs forming a finite set, there exist numbers $d, 0<d \leq \varepsilon$, and $L \geq 0$ such that $d_{D}\left(l_{k m}\right) \geq d$ and $\left|l_{k m}\right| \leq L$ for all $k, m=1, \ldots, N$. For every pair of points $z, w \in S$ there exist $z_{k}, z_{m}$ in the above $\varepsilon$-net with $\left|z-z_{k}\right|<\varepsilon$ and $\left|z_{m}-w\right|<\varepsilon$. Consequently, the arc $l(z, w)=\left[z, z_{k}\right] \cup l_{k m} \cup\left[z_{m}, w\right]$ joins $z$ and $w$ and has length of at most $L+2 \varepsilon \leq L+2$. By (2.8), applying Proposition 2.1(d3) twice, we obtain $\left[z, z_{k}\right] \cup\left[z_{m}, w\right] \subset D, d_{D}\left(\left[z, z_{k}\right]\right) \geq \varepsilon$, and $d_{D}\left(\left[z_{m}, w\right]\right) \geq \varepsilon$, i.e., $l(z, w) \subset D$. Since $0<d \leq \varepsilon \leq 1$, it follows that

$$
d_{D}(l(z, w)) \geq \min \left\{d, d_{D}\left(\left[z, z_{k}\right]\right), d_{D}\left(\left[z_{m}, w\right]\right)\right\} \geq \min \{d, \varepsilon\}=d .
$$

Therefore, $\frac{|l(z, w)|}{d_{D}(l(z, w))} \leq \frac{L+2}{d}$ for some arc $l(z, w) \subset D$ that joins $z$ and $w$, whence $\ell(S ; D) \leq \frac{L+2}{d}<+\infty$.

Conversely, let $\ell(S ; D)<+\infty$. Fix a point $z$ in $S$. Suppose that $S$ is not precompact in $D$. Then there exists a sequence $\left\{w_{n}\right\} \subset S, n=1,2, \ldots$, such that $w_{n} \rightarrow w \in \partial D$ as $n \rightarrow \infty$. By (2.15) , for every rectifiable arc $l\left(z, w_{n}\right) \subset D$ that joins $z$ and $w_{n}$ we obtain

$$
\frac{\left|l\left(z, w_{n}\right)\right|}{d_{D}\left(l\left(z, w_{n}\right)\right)} \geq \frac{\left|z-w_{n}\right|}{\operatorname{dist}\left(l\left(z, w_{n}\right), \partial D\right)} \geq \frac{\left|z-w_{n}\right|}{\operatorname{dist}\left(w_{n}, \partial D\right)} \geq \frac{\left|z-w_{n}\right|}{\left|w_{n}-w\right|} \underset{n \rightarrow \infty}{\longrightarrow}+\infty
$$

which contradicts the finiteness of $\ell(S ; D)$.

Remark. The definition of $\ell(S ; D)$ in the form (2.4d) can be extended also to unbounded domains $D \subset \mathbb{C}$. For this, it is most natural to pass to the spherical (chordal) distance on the extended complex plane. The entropy of arcwise connectedness can also be defined for subsets of domains in $\mathbb{R}^{n}$, or even for metric spaces, with preservation (to a certain extent) of properties mentioned in this section and further developed in Chapter III.

\section{§3. Elementary estimates involving the Harnack distance}

We remind the reader that the Harnack distance (see [45, 46]) in a domain $D$ is the function $\tau_{D}: D \times D \longrightarrow[1,+\infty)$ defined by the formula

$$
\tau_{D}(z, w)=\sup \{H(z) / H(w): H>0 \text { is harmonic in } D\} .
$$

In particular (see [45, Theorem 1.3.5]), for the disk $D(w, t)$ it is

$$
\tau_{D(w, t)}(z, w)=\frac{t+|z-w|}{t-|z-w|} .
$$

The function $\log \tau_{D}$ is a continuous semimetric10 on $D$ (see [45, Theorem 1.3.8]). In particular,

$$
\tau_{D}(z, w) \leq \tau_{D}(z, \zeta) \cdot \tau_{D}(\zeta, w), \quad z, \zeta, w \in D
$$

\footnotetext{
${ }^{10}$ If $D$ is a simply connected domain whose boundary contains at least two points, the function $(1 / 2) \log \tau_{D}(z, w)$ is none other than the hyperbolic distance on $D$ between $z$ and $w$.
} 
The Harnack distance obeys the subordination principle (see [45, Theorem 1.3.6]); in a weak form (see [45, Corollary 1.3.7]), it yields the implication

$$
D_{1} \subset D_{2} \Longrightarrow \tau_{D_{2}}(z, w) \leq \tau_{D_{1}}(z, w) \text {. }
$$

Proposition 3.1. Let $S$ be a subset of a domain D, and let

$$
\operatorname{diam} S<\operatorname{dist}(S, \partial D) \text {. }
$$

Then

$$
\sup _{z, w \in S} \tau_{D}(z, w) \leq 1+\frac{2 \operatorname{diam} S}{\operatorname{dist}(S, \partial D)-\operatorname{diam} S} .
$$

Proof. Let $z, w \in S$, and let $d=\operatorname{dist}(S, \partial D)$. Then $z \in D(w, d) \subset D$ by (3.5). Using the subordination principle (3.4) and the explicit form (3.2) of the Harnack distance in a disk, we deduce that

$$
\tau_{D}(z, w) \leq \tau_{D(w, d)}(z, w)=\frac{d+|z-w|}{d-|z-w|} \leq \frac{d+\operatorname{diam} S}{d-\operatorname{diam} S}=1+\frac{2 \operatorname{diam} S}{d-\operatorname{diam} S},
$$

and (3.6) follows.

The next estimate is also elementary and rather "vague", but involves the entropy of arcwise connectedness. It will be used for weighted algebras of holomorphic functions. A weaker version of it (not involving the entropy) was proved in [47, Proposition 2].

Proposition 3.2. Suppose that $S$ is a subset of a domain $D \Subset \mathbb{C}$. Then

$$
\sup _{z, w \in S} \tau_{D}(z, w) \leq 3^{2 \ell(S ; D)+1} .
$$

Proof. We may assume that $\ell(S ; D)$ is finite.

Let $z, w \in S$, and let $\ell(S ; D)<l<+\infty$. Consider a finite sequence of disks $D\left(\zeta_{k}, d\right) \subset$ $D, k=0, \ldots, n+1$, as in Proposition 2.2, so that conditions (D1) and (D2) are satisfied. By (3.3), we have $\tau_{D}(z, w) \leq \prod_{k=1}^{n+1} \tau_{D}\left(\zeta_{k-1}, \zeta_{k}\right)$. Since $D\left(\zeta_{k}, d\right) \subset D$, the subordination principle (3.4) applied to the right-hand side yields

$$
\begin{aligned}
\tau_{D}(z, w) & \leq \prod_{k=1}^{n+1} \tau_{D\left(\zeta_{k}, d\right)}\left(\zeta_{k-1}, \zeta_{k}\right) \\
& \stackrel{(3.2)}{=} \prod_{k=1}^{n+1} \frac{d+\left|\zeta_{k-1}-\zeta_{k}\right|}{d-\left|\zeta_{k-1}-\zeta_{k}\right|} \stackrel{(\mathrm{D} 2)}{\leq} \prod_{k=1}^{n+1} \frac{d+d / 2}{d-d / 2}=3^{n+1} .
\end{aligned}
$$

Now, to obtain (3.7), it suffices to employ the inequality $n \leq 2 l$ and the fact that $l>\ell(S ; D)$ can be taken arbitrarily.

\section{§4. Harmonic measure, Green's function, balayage, AND ESTIMATES OF INTEGRALS}

We recall some facts of potential theory on $\mathbb{C}$; see [38, 48, 49, 45].

Let $D \subset \mathbb{C}$ be a domain with nonpolar boundary. The symbols $\omega_{D}(z, \cdot)$ and $g_{D}(z, w)$ will denote (respectively) the harmonic measure for $D$ at the point $z$ and the extended Green function for $D$ (see [38, 5.7.4]) with pole at $z$. For each $z \in D$, the function $g_{D}(z, w)$ coincides with the Green function for $w \in D$, is subharmonic in the variable $w$ in $\mathbb{C} \backslash\{z\}$, is identically zero for $w \in \mathbb{C} \backslash \bar{D}$, and satisfies

$$
g_{D}\left(z, w_{0}\right)=\limsup _{\substack{w \rightarrow w_{0} \\ w \in D}} g_{D}(z, w), \quad w_{0} \in \partial D, z \in D .
$$

We note also that $g_{D}(z, w)$ is superharmonic in the variable $w$ for every fixed $z \in D$. 
We shall often use the classical Poisson-Jensen formula [38, Theorem 5.27]: if $v$ is subharmonic in a neighborhood of $\bar{D}$, then

$$
\int_{\partial D} v(w) d \omega_{D}(z, w)=v(z)+\int_{D_{i r}} g_{D}(z, w) d \mu_{v}(w), \quad z \in D
$$

where $D_{i r}$ is the union of $D$ and the set of all points of $\partial D$ irregular for the Dirichlet problem. It should be noted that in [38, Theorem 5.27] the integral on the left in the counterpart of (4.2) is taken over the set of regular points of $\partial D$ (rather than the entire set $\partial D$ ). But (4.2) is also true because the harmonic measure "does not feel" irregular points: $\omega_{D}\left(z, D_{i r}\right)=0, z \in D$.

Suppose a domain $D$ is precompact in $\Omega$. Among other things, (4.2) shows that, for $v \in S H(\Omega)$, the balayage

$$
\mathcal{H}_{D}^{v}(z) \stackrel{\text { def }}{=}\left\{\begin{array}{cl}
\int_{\partial D} v(w) d \omega_{D}(z, w) & \text { if } z \in D \\
v(z) & \text { if } z \in \Omega \backslash D
\end{array}\right.
$$

of $v$ from $D$ majorizes $v$ everywhere in $\Omega$, because the first line on the right in (4.3) is the harmonic extension of $v$ inside $D$. If $D$ is regular for the Dirichlet problem, then $\mathcal{H}_{D}^{p} \in S H(\Omega)$, i.e., this is the classical balayage 11 from $D$. In the general case, a balayage may fail to be subharmonic. For harmonic extension (consequently, for balayage), the subordination principle holds true:

$$
D_{1} \subset D_{2} \Subset \mathbb{C} \Longrightarrow \mathcal{H}_{D_{1}}^{v}(z) \leq \mathcal{H}_{D_{2}}^{v}(z), \quad z \in D_{1} .
$$

The subordination principle also applies to extended Green's functions:

$$
D_{1} \subset D_{2} \Subset \mathbb{C} \Longrightarrow g_{D_{1}}(z, w) \leq g_{D_{2}}(z, w), \quad z \in D_{1}, w \in \mathbb{C} .
$$

In the case where $z, w \in D_{1}$, this was observed, e.g., in [45, Corollary 4.4.5]; for $w \in \mathbb{C} \backslash \bar{D}_{1}$ the left-hand side of (4.5) is zero, and the right-hand side is nonnegative; for $w_{0} \in \partial D_{1}$, by (4.1) we have

$$
g_{D_{1}}\left(z, w_{0}\right) \leq \limsup _{\substack{w \rightarrow w_{0} \\ w \in D_{1}}} g_{D_{2}}(z, w) \leq g_{D_{2}}\left(z, w_{0}\right), \quad w_{0} \in \partial D_{1}, \quad z \in D_{1},
$$

where the last inequality follows from the fact that the function $g_{D_{2}}(z, w)$ is subharmonic (and upper semicontinuous) in $w$ for $w \neq z$.

Proposition 4.1. Let $V_{\mu}$ be a function subharmonic in a neighborhood of the closure $\bar{D}$ of a domain $D$, with Riesz measure $\mu$. Suppose $V$ is positive on $\bar{D}$. Furthermore, let $\lambda$ and $\nu$ be two measures in $\mathcal{M}^{+}(D)$ supported on a Borel set $S \Subset D$, i.e.,

$$
\lambda(D \backslash S)=\nu(D \backslash S)=0 .
$$

If

$$
\lambda(S) \leq \nu(S)
$$

then

$$
\int_{D} V_{\mu} d \lambda \leq \sup _{z, w \in S} \tau_{D}(z, w) \cdot\left(\int_{S} V_{\mu}(w) d \nu(w)+\int_{D_{i r}} \int_{S} g_{D}(w, \zeta) d \nu(w) d \mu(\zeta)\right) .
$$

Moreover, if $0 \notin \bar{D} \Subset \Omega$, where $\Omega$ is a domain in $\mathbb{C}, 0 \in \Omega$, and $V_{\mu}$ is the potential of a Jensen measure $\mu \in J_{0}(\Omega)$, then the outer integral in the last summand in (4.8) can be taken over $D$.

\footnotetext{
${ }^{11}$ Also (see, e.g., 48,49$]$ ), this function is denoted by $\mathcal{B}_{p}^{\Omega \backslash D}$ and called a function swept out with respect to $p$ and the set $\Omega \backslash D$.
} 
Proof. The harmonic extension $\mathcal{H}_{D}^{V}(w)$ of $V=V_{\mu}$ inside $D$ majorizes $V$ on $D$; since $V$ is positive on $\partial D$, this extension is positive on $D$. By the definition (3.1) of the Harnack distance, it follows that

$$
V(z) \leq \mathcal{H}_{D}^{V}(z) \leq \tau_{D}(z, w) \mathcal{H}_{D}^{V}(w)=\tau_{D}(z, w) \int_{\partial D} V(\zeta) d \omega_{D}(w, \zeta), z, w \in D .
$$

To the integral on the right in (4.2), we apply the Poisson-Jensen formula (4.9) to obtain

$$
V(z) \leq \tau_{D}(z, w)\left(V(w)+\int_{D_{i r}} g_{D}(w, \zeta) d \mu(\zeta)\right), \quad z, w \in D .
$$

Since $V$ and $g_{D}$ are positive on $\bar{D}$, from the above formula we deduce that

$$
\begin{aligned}
\int_{D} V d \lambda \stackrel{\text { 4.6) }}{\leq} \sup _{z \in S} V(z) \cdot \lambda(S) \\
\stackrel{\text { 4.10 }}{\leq} \sup _{z, w \in S} \tau_{D}(z, w) \cdot\left(V(w)+\int_{D_{i r}} g_{D}(w, \zeta) d \mu(\zeta)\right) \cdot \nu(S), \quad w \in S .
\end{aligned}
$$

This being true for all $w \in S$, we infer (also taking (4.6) into account) that

$$
\int_{D} V d \lambda \leq \sup _{z, w \in S} \tau_{D}(z, w) \cdot \int_{S}\left(V(w)+\int_{D_{i r}} g_{D}(w, \zeta) d \mu(\zeta)\right) d \nu(w),
$$

which is (4.8) (it suffices to use the Fubini theorem as it is presented in [38, Theorem 3.5]).

The final part of Proposition 4.1 follows from the fact that a Jensen measure $\mu \in J_{0}(\Omega)$ takes the value 0 at each set of zero outer capacity not containing 0 , and the set of irregular points has these properties.

\section{§5. Stars of SETS, And BALAYAGE}

The terminology used in what follows agrees with that in [50, Chapter V].

Let $\Omega$ be a domain in $\mathbb{C}$, and $\mathcal{D}$ a family of subsets of $\Omega$. The boundary $\partial \mathcal{D}$ of $\mathcal{D}$ is defined to be the union of the boundaries (in $\Omega$ ) of all members of $\mathcal{D}$.

The family $\mathcal{D}$ is said to be separated from a point $z \in \Omega$ if some neighborhood of this point in $\Omega$ does not intersect any member of $\mathcal{D}$. The family $\mathcal{D}$ is said to be locally finite in $\Omega$ if every $z \in \Omega$ has a neighborhood that intersects only finitely many members of $\mathcal{D}$. Clearly, a locally finite family is at most countable (in our situation), and every precompact subset of $\Omega$ intersects at most finitely many sets in this family (50, Problem 38]). An indexed family ${ }^{12} \mathcal{D}=\left\{D_{k}\right\}, k=1,2, \ldots$, is combinatorially inscribed in an indexed family $\mathcal{D}^{\prime}=\left\{D_{k}^{\prime}\right\}, k=1,2, \ldots$, if $D_{k} \subset D_{k}^{\prime}$ for all $k$.

Definition 5.1. A family $\mathcal{D}$ of domains included in a domain $\Omega$ is said to be admissible for $\Omega$ if the following three conditions are satisfied:

(1) every domain in $\mathcal{D}$ is precompact in $\Omega$;

(2) $\mathcal{D}$ is locally finite;

(3) every domain in $\mathcal{D}$ is squarable with respect to the Lebesgue measure $m$, i.e., $m(\partial D)$ $=0$ for all $D \in \mathcal{D}$.

Conditions (2) and (3) imply that the boundary $\partial \mathcal{D}$ of a family $\Omega$ admissible for $\mathcal{D}$ has Lebesgue measure zero. We note in passing that for every locally finite family $\Sigma=\left\{S_{k}\right\}$, $k=1,2, \ldots$, where $S_{k} \Subset \Omega$, the family $\bar{\Sigma}=\left\{\bar{S}_{k}\right\}$ of compact subsets of $\Omega$ is also locally finite (see [50, Problem 37]), and it is easy to find an admissible family $\mathcal{D}=\left\{D_{k}\right\}$ of domains in $\Omega$ such that $\bar{\Sigma}$ is inscribed combinatorially in $\mathcal{D}$.

\footnotetext{
${ }^{12}$ When talking about an indexed family, we do not demand that its elements be pairwise different.
} 
The star of a set $A \subset \Omega$ relative to a family $\mathcal{D}$ is defined to be the set

$$
\mathcal{D}_{\star}(A) \stackrel{\text { def }}{=} \bigcup\{D \in \mathcal{D}: A \cap D \neq \varnothing\} \subset \Omega .
$$

The star of a point $z \in \Omega$ relative to $\mathcal{D}$ is the set

$$
\mathcal{D}_{\star}(z) \stackrel{\text { def }}{=} \mathcal{D}_{\star}(\{z\})=\bigcup\{D \in \mathcal{D}: z \in D\} \subset \Omega .
$$

The family $\mathcal{D}_{\star}=\left\{\mathcal{D}_{\star}(z): z \in \Omega\right\}$ is called the family of stars for $\mathcal{D}$ in $\Omega$. Clearly, if $\mathcal{D}$ is a family of domains in $\Omega$, then so is $\mathcal{D}_{\star}$; if $\mathcal{D}$ is separated from a point, $\mathcal{D}_{\star}$ is also separated from the same point. Moreover, if $\mathcal{D}$ is an admissible family, then:

(a) $\partial \mathcal{D}_{\star}$ is of Lebesgue measure zero;

(b) $\mathcal{D}_{\star}(z)$ is precompact in $\Omega$ for every $z \in \Omega$;

(c) the family $\mathcal{D}$ is locally constant outside the boundary $\partial \mathcal{D}$, i.e., every $z \in \Omega \backslash \partial \mathcal{D}$ possesses a neighborhood (in $\Omega$ ) such that the stars of all points of this neighborhood relative to $\mathcal{D}$ coincide.

Let $g_{\mathcal{D}_{\star}(\zeta)}(w, \zeta)$ be the evaluation at $\zeta$ (in the second argument) of the extended Green function for $\mathcal{D}_{\star}(\zeta)$ if $\mathcal{D}_{\star}(\zeta)$ is nonempty, and let $g_{\mathcal{D}_{\star}(\zeta)}(w, \zeta) \equiv 0$ otherwise.

Proposition 5.1. Let $\mathcal{D}=\left\{D_{k}\right\}$ be an admissible family of domains in $\Omega$, and let $\nu^{(k)}$ be a measure with support in one of the $D_{k}$. Furthermore, let $p \not \equiv-\infty$ be a subharmonic function in $\Omega$. Then the functions

$$
\int_{\mathcal{D}_{\star}(\zeta)} g_{\mathcal{D}_{\star}(\zeta)}(w, \zeta) d \nu^{(k)}(w), \quad \zeta \in \Omega,
$$

and $\mathcal{H}_{\mathcal{D}_{\star}(\zeta)}^{p}(\zeta)$ are measurable and locally integrable with respect to the Lebesgue measure $m$ on $\Omega$, and also with respect to every Jensen measure $\mu$ that is $m$-absolutely continuous.

Proof. By properties (b) and (c) of the family $\mathcal{D}_{\star}$ constructed for an admissible family $\mathcal{D}$, it is easily seen that, as a function of $\zeta \in \Omega$, the integral (5.3) is locally superharmonic off the boundary $\partial \mathcal{D}$ (because so is the Green function $g_{\mathcal{D}_{\star}(\zeta)}(w, \zeta)$ ). Consequently, (5.3) represents a measurable and Lebesgue integrable function of $\zeta$ (because $m(\partial \mathcal{D})=0$ ). Again in view of properties (b) and (c), it easily follows that $\mathcal{H}_{\mathcal{D}_{\star}(\zeta)}^{p}(\zeta)$ is a locally subharmonic function of $\zeta \in \Omega$ off the boundary $\partial \mathcal{D}$. In particular, it is measurable and Lebesgue locally integrable, because $m(\partial \mathcal{D})=0$. The final statement on Jensen's measures is obvious.

The star with kernel for a set $A \subset \Omega$ relative to a family $\mathcal{D}$ is defined to be the set

$$
\mathcal{D}_{\star}[A] \stackrel{\text { def }}{=} A \cup \mathcal{D}_{\star}(A)=A \cup(\bigcup\{D \in \mathcal{D}: A \cap D \neq \varnothing\}) \subset \Omega .
$$

If $A$ intersects no member of $\Omega$, then $\mathcal{D}_{\star}(A)=\varnothing$, but $\mathcal{D}_{\star}[A]=A$.

If $A$ is a singleton $\{z\}$ or a disk $D(z, t)$ in (5.4), we introduce a short notation for the corresponding stars with kernel:

$$
\mathcal{D}_{\star}[z] \stackrel{\text { def }}{=} \mathcal{D}_{\star}[\{z\}]=\{z\} \cup \mathcal{D}_{\star}(z), \quad \mathcal{D}_{\star}[z, t) \stackrel{\text { def }}{=} \mathcal{D}_{\star}[D(z, t)] .
$$

Suppose a function $r$ on $\Omega$ satisfies (1.3). For a set $A \subset \Omega$, we define its $r$-blowup in $\Omega$ (compare with (2.2) ) as follows:

$$
A^{r} \stackrel{\text { def }}{=} \bigcup\{D(z, r(z)): z \in A\} .
$$

If $A$ is connected, this is a subdomain of $\Omega$.

In the rest of this section, we consider only admissible families $\mathcal{D}$ of domains in $\Omega$. 
Proposition 5.2. If $r, r_{0}: \Omega \rightarrow \mathbb{R}$ are functions satisfying (1.3) and $r \leq r_{0}$ on $\Omega$, then

$$
\mathcal{H}_{\left(\mathcal{D}_{\star}[z, t)\right)^{r}}^{p}(z) \leq \mathcal{H}_{\left(\mathcal{D}_{\star}[z, t)\right)^{r_{0}}}^{p}(z), \quad p \in S H(\Omega),
$$

for $z \in \Omega$ and $D(z, t) \Subset \Omega$.

Since $\left(\mathcal{D}_{\star}[z, t)\right)^{r} \subset\left(\mathcal{D}_{\star}[z, t)\right)^{r_{0}}$ for $r \leq r_{0}$, the above statement is an immediate consequence of the subordination principle (4.4) for balayage.

The following proposition will be used to rewrite some estimates in a more compact form.

Proposition 5.3. Suppose $r$ is a continuous function on $\Omega$ satisfying (1.3). If $p \in$ $S H(\Omega)$, then

$$
\begin{gathered}
p^{(r)}(z) \leq \mathcal{H}_{\left(\mathcal{D}_{\star}[z, t)\right)^{r}}^{p}(z), \\
\frac{1}{2 \pi} \int_{0}^{2 \pi}\left(\mathcal{H}_{\mathcal{D}_{\star}\left(z+t e^{i \theta}\right)}^{p}\right)^{(r)}\left(z+t e^{i \theta}\right) d \theta \leq \mathcal{H}_{\left(\mathcal{D}_{\star}[z, t)\right)^{r}}^{p}(z)
\end{gathered}
$$

for all $z \in \Omega$ and all $t$ with $0<t<\operatorname{dist}(z, \partial \Omega)$.

Proof. By the construction (4.3) of balayage, the right-hand side of (5.8a) is a harmonic majorant of $p$ in the domain $\left(\mathcal{D}_{\star}[z, t)\right)^{r}$; this domain includes the disk $D(z, r(z))$. Therefore, averaging (as in (1.4)) the function $p$ and the right-hand side in (5.8a) over the disk $D(z, r(z))$ implies (5.8a).

By (1.3) and the continuity of $r$, we have $\mathcal{D}_{\star}\left(z+t e^{i \theta}\right) \subset\left(\mathcal{D}_{\star}[z, t)\right)^{r}$. By the subordination principle (4.4) for balayage, we obtain $\mathcal{H}_{\mathcal{D}_{*}\left(z+t e^{i \theta}\right)}^{p}(\zeta) \leq \mathcal{H}_{\left(\mathcal{D}_{\star}[z, t)\right)^{r}}^{p}(\zeta), \zeta \in \Omega$, where on the right we have a harmonic function in $\left(\mathcal{D}_{\star}[z, t)\right)^{r}$. Taking this into account and averaging (as in (1.4) ) both sides of the above inequality over the disks $D\left(z+t e^{i \theta}, r\left(z+t e^{i \theta}\right)\right)$, we arrive at

$$
\left(\mathcal{H}_{\mathcal{D}_{\star}\left(z+t e^{i \theta}\right)}^{p}\right)^{(r)}\left(z+t e^{i \theta}\right) \leq \mathcal{H}_{\left(\mathcal{D}_{\star}[z, t)\right)^{r}}^{p}\left(z+t e^{i \theta}\right), \quad \theta \in[0,2 \pi] .
$$

Since the right-hand side of this inequality is harmonic in a neighborhood of the closure of $D(z, t)$, averaging both sides over the circle $\partial D(z, t)$ yields $(5.8 \mathrm{~b})$.

The next statement is a version of Lemma 1.1

Proposition 5.4. Suppose $r \in C(\Omega)$ satisfies (1.3), and $v \in S H(\Omega), v \not \equiv-\infty$. Then there exists a function $h \in H(\Omega), h(0) \neq 0$, such that

$$
\log |h(z)| \leq \mathcal{H}_{\left(\mathcal{D}_{\star}[z, t)\right)^{r}}^{v}(z)+\log \left(1+\frac{1}{t}\right)+9 \log (1+|z|)
$$

whenever $z \in \Omega$ and $0<t<\operatorname{dist}(z, \partial \Omega)$.

Proof. Let $U$ be a neighborhood of zero such that $U \Subset \Omega$. We put $3 \beta=\min \{r(z)$ : $z \in \bar{U}\}>0$. Consider the balayage $v_{\beta}=\mathcal{H}_{D(3 \beta)}^{v}$ of $v$ from the disk $D(3 \beta)$. The function $v_{\beta}$ is subharmonic and, by the choice of $\beta$, satisfies (1.11); moreover, $v_{\beta} \leq v^{(r)}$ on $\Omega$. In view of Lemma 1.1, there exists a function $h \in H(\Omega), h(0) \neq 0$, such that

$$
\log |h(z)| \leq \frac{1}{2 \pi} \int_{0}^{2 \pi} v^{(r)}\left(z+t e^{i \theta}\right) d \theta+\log \left(1+\frac{1}{t}\right)+9 \log (1+|z|), \quad z \in \Omega,
$$

for $0<t<\operatorname{dist}(z, \partial \Omega)$. By the definition of balayage, we have $v^{(r)}(\zeta) \leq\left(\mathcal{H}_{\mathcal{D}_{\star}(\zeta)}^{v}\right)^{(r)}(\zeta)$ for $\zeta \in \Omega$. Together with (5.10) and (5.8b), this implies (5.9). 


\section{§6. Auxiliary Estimates of integrals in terms of SPlitting of MeAsures}

Propositions 6.1 and 6.2 below generalize (the main) Lemma 1.1 in [23] (see also [24, Comparison lemma]), which pertains to the case of $\Omega=\mathbb{C}$.

Proposition 6.1. Suppose $\Omega$ is a domain in $\mathbb{C}$ and $0 \in \Omega$. Suppose also that positive measures $\lambda$ and $\nu$ on $\Omega$ are represented as sums

$$
\lambda=\sum_{k=1}^{\infty} \lambda^{(k)}, \quad \nu=\sum_{k=1}^{\infty} \nu^{(k)},
$$

of positive measures supported on Borel sets $S_{k}$ that are precompact in $\Omega$, i.e.,

$$
\lambda^{(k)}\left(\Omega \backslash S_{k}\right)=\nu^{(k)}\left(\Omega \backslash S_{k}\right)=0, \quad S_{k} \Subset \Omega, \quad k=1,2, \ldots .
$$

If the family $S_{k}$ is combinatorially inscribed in a family $\mathcal{D}=\left\{D_{k}\right\}, k=1,2, \ldots$, that is admissible for $\Omega$ and separated from zero, and

$$
\lambda^{(k)}\left(S_{k}\right) \leq \nu^{(k)}\left(S_{k}\right), \quad k=1,2, \ldots,
$$

then for every Jensen measure $\mu \in J_{0}(\Omega)$ absolutely continuous with respect to $m$ we have

$$
\begin{aligned}
\int_{\Omega} V_{\mu} d \lambda \leq & \sum_{k=1}^{\infty} \sup _{z, w \in S_{k}} \tau_{D_{k}}(z, w) \\
& \times\left(\int_{\Omega} V_{\mu} d \nu^{(k)}+\int_{\Omega} \int_{\mathcal{D}_{\star}(\zeta)} g_{\mathcal{D}_{\star}(\zeta)}(w, \zeta) d \nu^{(k)}(w) d \mu(\zeta)\right),
\end{aligned}
$$

where $V_{\mu}$ is the potential of $\mu$.

Proof. Relations (6.2) and (6.3) imply (4.6) and (4.7) (with $S=S_{k}, D=D_{k}, \lambda=\lambda^{(k)}$ and $\left.\nu=\nu^{(k)}\right)$; so, we can apply Proposition 4.1 to obtain

$$
\begin{aligned}
& \int_{D_{k}} V_{\mu} d \lambda^{(k)} \leq \sup _{z, w \in S_{k}} \tau_{D_{k}}(z, w) \\
& \times\left(\int_{S_{k}} V_{\mu}(w) d \nu^{(k)}(w)+\int_{D_{k}} \int_{S_{k}} g_{D_{k}}(w, \zeta) d \nu^{(k)}(w) d \mu(\zeta)\right), \\
& k=1,2, \ldots
\end{aligned}
$$

The inner integral in the last term in (6.5) can only become greater if we replace $g_{D_{k}}(w, \zeta)$, $\zeta \in \mathbb{C}$, with $g_{\mathcal{D}_{\star}(\zeta)}(w, \zeta), \zeta \in \mathbb{C}$ (the latter function majorizes the former by the subordination principle (4.5) ) and enlarge the domain $S_{k}$ of integration to $\mathcal{D}_{\star}(\zeta) \supset D_{k} \supset S_{k}$, $\zeta \in D_{k}$. Thus, the inner integral does not exceed the integral (6.5). By Proposition 5.1, we can plug (5.3) in (6.5); then we extend the outer integration to $\Omega$. Summation over $k$ yields (6.4).

Proposition 6.2. Let $p$ be a subharmonic function in a domain $\Omega \ni 0$ with Riesz measure $\nu, 0 \notin \operatorname{supp} \nu$. Suppose an admissible family $\mathcal{D}=\left\{D_{k}\right\}$ of domains in $\Omega$ is separated from zero. Then for every Jensen measure $\mu \in J_{0}(\Omega) \cap \mathcal{M}_{\mathrm{ac}}^{+}(\Omega)$ with potential $V_{\mu}$ we have

$$
\int_{\Omega} V_{\mu} d \nu+\int_{\Omega} \int_{\mathcal{D}_{\star}(\zeta)} g_{\mathcal{D}_{\star}(\zeta)}(w, \zeta) d \nu(w) d \mu(\zeta) \leq \int_{\Omega} \mathcal{H}_{\mathcal{D}_{\star}(\zeta)}^{p}(\zeta) d \mu(\zeta)-p(0),
$$

where, clearly, $p(0) \neq-\infty$ because $0 \notin \operatorname{supp} \nu$, and the right-hand side is positive.

Proof. By (1.6) in Proposition 1.1, for the first integral in (6.6) we have

$$
\int_{\Omega} V_{\mu} d \nu=\int_{\Omega} p(\zeta) d \mu(\zeta)-p(0)
$$


Using this, we rewrite the left-hand side of (6.7) in the form

$$
\int_{\Omega}\left(p(\zeta)+\int_{\mathcal{D}_{\star}(\zeta)} g_{\mathcal{D}_{\star}(\zeta)}(w, \zeta) d \nu(w)\right) d \mu(\zeta)-p(0) .
$$

If $\mathcal{D}_{\star}(\zeta) \neq \varnothing$, then $\zeta \in \mathcal{D}_{\star}(\zeta)$ by the construction (5.2) of the star of $\zeta$. Then the classical Poisson-Jensen formula (4.2) yields the following estimate for the integrand with respect to $\mu$ in (6.8):

$$
p(\zeta)+\int_{\mathcal{D}_{\star}(\zeta)} g_{\mathcal{D}_{\star}(\zeta)}(w, \zeta) d \nu(w) \leq \int_{\partial \mathcal{D}_{\star}(\zeta)} p(w) d \omega_{\mathcal{D}_{\star}(\zeta)}(\zeta, w) .
$$

The right-hand side in (6.9) is the boundary value at $\zeta$ of the harmonic extension $\mathcal{H}_{\mathcal{D}_{\star}(\zeta)}^{p}$ of $p$ to $\mathcal{D}_{\star}(\zeta)$. But if $\mathcal{D}_{\star}(\zeta)=\varnothing$, then the integral on the left in (6.9) is zero, and only $p(\zeta)$ remains there. Thus, in the notation (4.3), the left-hand side of (6.9) is dominated by the balayage $\mathcal{H}_{\mathcal{D}_{\star}(\zeta)}^{p}(\zeta)$ and, in view of Proposition [5.1, (6.8) does not exceed $\int_{\Omega} \mathcal{H}_{\mathcal{D}_{\star}(\zeta)}^{p}(\zeta) d \mu(\zeta)-p(0)$, as required.

\section{Chapter II. Preparatory theorems}

\section{§7. Preparatory theorem for algebras}

Throughout this chapter,

(i) $u$ and $p$ are subharmonic functions in a domain $\Omega \ni 0$, with Riesz measures $\lambda_{u}$ and $\nu_{p}$

(ii) a family $\Sigma=\left\{S_{k}\right\}$ of Borel sets $S_{k} \Subset \Omega, k=1,2, \ldots$, is combinatorially inscribed in an admissible family of domains $\mathcal{D}=\left\{D_{k}\right\}$ in $\Omega$;

(iii) the measures $\lambda_{u}$ and $\nu_{p}$ are representable as sums of positive measures (cf. (6.1))

$$
\lambda_{u}=\lambda^{(0)}+\sum_{k=1}^{\infty} \lambda^{(k)}, \quad \nu_{p}=\nu^{(0)}+\sum_{k=1}^{\infty} \nu^{(k)},
$$

where $\lambda^{(0)}$ is supported on a compact subset of $\Omega$, and $\lambda^{(k)}, \nu^{(k)}$ are supported on $S_{k}$, i.e., (6.2) is fulfilled.

A function $r \geq 0$ on $\Omega$ is said to be locally separated from zero if every $z \in \Omega$ has a neighborhood $U_{z} \subset \Omega$ with $\inf _{\zeta \in U_{z}} r(\zeta)>0$.

Theorem 7.1 (Preparatory theorem). Under conditions (ii)-(iii), suppose that 13

$$
\begin{gathered}
\limsup _{k \rightarrow \infty} \ell\left(S_{k} ; D_{k}\right)=\bar{l}<+\infty, \\
\limsup _{k \rightarrow \infty} \frac{\lambda^{(k)}\left(S_{k}\right)}{\nu^{(k)}\left(S_{k}\right)}=\bar{s}<+\infty .
\end{gathered}
$$

Then for every $l>\bar{l}$ and $s>\bar{s}$ and every function

$$
r: \Omega \rightarrow \mathbb{R}, \quad 0<r(z)<d_{\Omega}(z) \stackrel{\text { def }}{=} \operatorname{dist}(z, \partial \Omega), \quad z \in \Omega,
$$

locally separated from zero, there exists a holomorphic function $h \not \equiv 0$ in $\Omega$ that, in the notation (4.3) and (5.4)-(5.6), satisfies

$$
u(z)+\log |h(z)| \leq \inf _{0<t<d_{\Omega}(z)}\left(A \mathcal{H}_{\left(\mathcal{D}_{\star}[z, t)\right)^{r}}^{p}(z)+\log (1+1 / t)\right)+B \log (1+|z|)
$$

for all $z \in \Omega$, where $B \geq 0$ is some constant and $A=3^{2 l+1} s$.

\footnotetext{
${ }^{13}$ See Definition 2.1 of the entropy $\ell\left(S_{k} ; D_{k}\right)$ of arcwise connectedness.
} 
Proof. By (7.21 $)$ and (7.21 $)$, there exists $k_{1}$ such that for all $k \geq k_{1}$ we have

$$
\begin{aligned}
& \ell\left(S_{k}, \partial D_{k}\right) \leq l, \\
& \lambda^{(k)}\left(S_{k}\right) \leq s \nu^{(k)}\left(S_{k}\right) .
\end{aligned}
$$

We take a sufficiently small $\varepsilon>0$ such that $D(\varepsilon) \Subset \Omega$ and put

$$
N=\max \left\{k \in \mathbb{N}: k<k_{1} \text { or } D_{k} \cap D(\varepsilon) \neq \varnothing\right\} .
$$

We redenote $\lambda^{(0)}+\sum_{k=1}^{N} \lambda^{(k)}$ and $\nu^{(0)}+\sum_{k=1}^{N} \nu^{(k)}$ by $\lambda^{(0)}$ and $\nu^{(0)}$, and the "truncated" families $\left\{S_{k}\right\}_{k>N}$ and $\left\{D_{k}\right\}_{k>N}$ by $\Sigma$ and $\mathcal{D}$; moreover, we renumber the members of $\Sigma$ and $\mathcal{D}$ by all the positive integers (starting from 1 ) so that $\Sigma$ is combinatorially inscribed in $\mathcal{D}$, as before. Clearly, (ii)-(iii) are preserved in the new notation, $\mathcal{D}$ is separated from zero, and relations (7.5) become valid for all $k \in \mathbb{N}$.

Now, we represent $u$ and $p$ in the form

$$
u=u_{0}+u_{\infty}, \quad p=p_{0}+p_{\infty},
$$

where

$$
u_{0}(z)=\int_{\Omega} \log |z-w| d \lambda^{(0)}(w)
$$

and $p_{0}$ are subharmonic functions whose Riesz measures are $\lambda^{(0)}$ and $\nu^{(0)}$. Taking the above renumbering into account, we see that the measures

$$
\lambda_{\infty} \stackrel{\text { def }}{=} \sum_{k=1}^{\infty} \lambda^{(k)}, \quad \nu_{\infty} \stackrel{\text { def }}{=} \sum_{k=1}^{\infty} \nu^{(k)}
$$

are Riesz measures for $u_{\infty}$ and $p_{\infty}$. By (3.7) in Proposition 3.2 and (7.51]), we obtain

$$
\sup _{z, w \in S_{k}} \tau_{D_{k}}(z, w) \leq 3^{2 \ell\left(S_{k}, D_{k}\right)+1} \leq 3^{2 l+1}=c .
$$

We apply Proposition 6.1 to the pairs of measures $\lambda^{(k)}, s \nu^{(k)}, k=1,2 \ldots$ The second components $s \nu^{(k)}$ of each pair play the same role as the measures $\nu^{(k)}$ in (6.1), (6.2), and (6.3); now we take $s p_{\infty}$ (with Riesz measure $s \nu_{\infty}$ ) for the role of $p$. Since inequality (7.5) is fulfilled, we can apply Proposition 6.1. So, combining (6.4) and (7.10), we see that

$$
\begin{aligned}
\int_{\Omega} V_{\mu} d \lambda_{\infty} & \leq c \sum_{k=1}^{\infty}\left(\int_{\Omega} V_{\mu} d\left(s \nu^{(k)}\right)+\int_{\Omega} \int_{\mathcal{D}_{\star}(\zeta)} g_{\mathcal{D}_{\star}(\zeta)}(w, \zeta) d\left(s \nu^{(k)}\right)(w) d \mu(\zeta)\right) \\
& =c s\left(\int_{\Omega} V_{\mu} d \nu_{\infty}+\int_{\Omega} \int_{\mathcal{D}_{\star}(\zeta)} g_{\mathcal{D}_{\star}(\zeta)}(w, \zeta) d \nu_{\infty}(w) d \mu(\zeta)\right)
\end{aligned}
$$

for every Jensen measure $\mu \in J_{0}(\Omega) \cap \mathcal{M}_{\mathrm{ac}}^{+}(\Omega)$ with potential $V_{\mu}$. Using (6.6) (with substitution of $p_{\infty}$ for $p$ ) to estimate the last term in parentheses, we arrive at the estimate

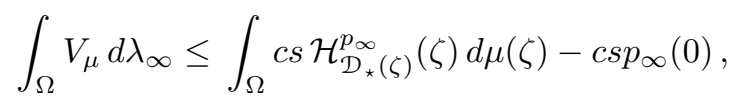

where $p_{\infty}(0) \neq-\infty$ because $0 \notin \operatorname{supp} \nu_{\infty}$ (the family $\mathcal{D}$ is separated from zero).

Formula (7.11) means that the assumption (1.7) of Theorem 1.2 is fulfilled for the function $M(\zeta)=c s \mathcal{H}_{\mathcal{D}_{\star}(\zeta)}^{p_{\infty}}(\zeta)$ (which is locally integrable with respect to $m$ and $\mu$; see Proposition 5.1) and the constant $C=-c s p_{\infty}(0)$. 
Assume for the moment that the function $r>0$ (satisfying (7.3)) is continuous. By Theorem 1.2 there exists $h_{\infty} \in H(\Omega), h_{\infty} \not \equiv 0$, for which an estimate of the form (7.4) is fulfilled with $A=c s=3^{2 l+1} s$ ( $c$ is from (7.10) $)$, namely,

$$
\begin{gathered}
u_{\infty}(z)+\log \left|h_{\infty}(z)\right| \\
\leq A \frac{1}{2 \pi} \int_{0}^{2 \pi}\left(\mathcal{H}_{\mathcal{D}_{\star}\left(z+t e^{i \theta}\right)}^{p_{\infty}}\right)^{(r)}\left(z+t e^{i \theta}\right) d \theta+\log (1+1 / t)+9 \log (1+|z|) \\
\frac{5.8 \mathrm{~b}}{\leq} A \mathcal{H}_{\left(\mathcal{D}_{\star}[z, t)\right)^{r}}^{p_{\infty}}(z)+\log (1+1 / t)+9 \log (1+|z|), \\
z \in \Omega, \quad 0<t<\operatorname{dist}(z, \partial \Omega) .
\end{gathered}
$$

By the construction (7.8) of $u_{0}$, we have (see [51, 6.7.2])

$$
u_{0}(z) \leq \lambda^{(0)}(\Omega) \log (1+|z|)+C .
$$

By Proposition 5.4 applied to $v=A p_{0}$, there exists a function $h_{0} \not \equiv 0$ in $\Omega$ such that

$$
\log \left|h_{0}(z)\right| \leq A \mathcal{H}_{\left(\mathcal{D}_{\star}[z, t)\right)^{r}}^{p_{0}}(z)+\log \left(1+\frac{1}{t}\right)+9 \log (1+|z|)
$$

for every $z \in \Omega$ and $0<t<\operatorname{dist}(z, \partial \Omega)$. Adding (7.12), (7.13), and (7.14) and using (7.7), we obtain (7.4) with $h=h_{\infty} h_{0} \exp (-C)$ and $B=18+\lambda^{(0)}(\Omega)$.

The following elementary lemma allows us to lift the assumption of continuity for $r$.

Lemma 7.1. For every function $r_{0} \geq 0$ separated from zero on $\Omega$, there exists $r \in C(\Omega)$ such that $0<r(z) \leq r_{0}(z)$ for $z \in \Omega$.

To finish the proof of the theorem, it suffices to combine Lemma 7.1. inequality (5.7) in Proposition 5.2, and the special case of the theorem established above.

Remark. The freedom in the choice of $t$ in (17.4) leads to various estimates. Thus, putting $t=t(z)=r(z)$ in (7.4), we arrive at

$$
u(z)+\log |h(z)| \leq A \mathcal{H}_{\left(\mathcal{D}_{\star}[z, r(z))\right)^{r}}^{p}(z)+\gamma \log \left(1+\frac{1}{r(z)}\right)+B \log (1+|z|), \quad z \in \Omega
$$

In any case, the values of $l>\bar{l}$ and $s>\bar{s}$ can be chosen so as to make the constant $A=3^{2 l+1} s$ in (7.4) a natural number.

\section{§8. Preparatory theorems for spaces of holomorphic FunCtions}

The next theorem will be applied to spaces of the form $H_{p}^{1}(\Omega)$.

Theorem 8.1 (Preparatory theorem). Under conditions (ii)-(iii) in $\S 7$, suppose that

$$
\begin{gathered}
\limsup _{k \rightarrow \infty} \frac{\operatorname{diam} S_{k}}{\operatorname{dist}\left(S_{k}, \partial D_{k}\right)}<\alpha<1, \\
\limsup _{k \rightarrow \infty} \frac{\lambda^{(k)}\left(S_{k}\right)}{\nu^{(k)}\left(S_{k}\right)}<\beta
\end{gathered}
$$

for some $\alpha>0$ and $\beta>0$. Then for every function $r$ satisfying (7.3) and locally separated from zero, there exists a function $h \in H(\Omega), h \neq 0$, and a constant $B \geq 0$ such that, in the notation (4.3) and (5.4) (5.6), we have

(8.2) $u(z)+\log |h(z)| \leq \inf _{0<t<d_{\Omega}(z)}\left(\frac{1+\alpha}{1-\alpha} \beta \mathcal{H}_{\left(\mathcal{D}_{\star}[z, t)\right)^{r}}^{p}(z)+\log \left(1+\frac{1}{t}\right)\right)+B \log (1+|z|)$

for all $z \in \Omega$. 
Proof. As in the proof of the preceding theorem, taking Lemma 7.1 and (5.7) in Proposition 5.2 into account, it suffices to consider the case where $r$ is a continuous function.

By (8.1), for some $k_{1} \in \mathbb{N}$ we have

$$
\operatorname{diam} S_{k} \leq \alpha \operatorname{dist}\left(S_{k}, \partial D_{k}\right)
$$

$$
\lambda^{(k)}\left(S_{k}\right) \leq \beta \nu^{(k)}\left(S_{k}\right)
$$

for all $k \geq k_{1}$. In particular, (8.31] implies that

$$
1+\frac{2 \operatorname{diam} S_{k}}{\operatorname{dist}\left(S_{k}, \partial D_{k}\right)-\operatorname{diam} S_{k}} \leq \frac{1+\alpha}{1-\alpha}
$$

for $k \geq k_{1}$.

We choose a number $N$ as in (7.6) and pass to the representation (7.7), as at the beginning of the proof of Theorem 7.1. Using (7.13) and (7.14), it is easy eliminate the influence of $u_{0}$ and $p_{0}$. Therefore, from now on we may assume without loss of generality that the family $\mathcal{D}$ is separated from zero, inequalities (8.38) and (8.4) are true for all $k \in \mathbb{N}$, and there are no measures $\lambda^{(0)}$ and $\nu^{(0)}$ in (7.1). After these simplifications, by (8.4) and Proposition 3.1, estimate (3.6) implies

$$
\sup _{z, w \in S_{k}} \tau_{D_{k}}(z, w) \leq \frac{1+\alpha}{1-\alpha}, \quad k=1,2, \ldots
$$

Inequalities (8.38) coincide with (6.3) if we substitute $\beta \nu$ for $\nu$. Consequently, for every Lebesgue absolutely continuous Jensen measure $\mu \in J_{0}(\Omega)$ (with potential $V_{\mu}$ ), Proposition 6.1 and 8.5 yield

$$
\begin{aligned}
\int_{\Omega} V_{\mu} d \lambda & \leq \frac{1+\alpha}{1-\alpha} \sum_{k=1}^{\infty}\left(\int_{\Omega} V_{\mu} d\left(\beta \nu^{(k)}\right)+\int_{\Omega} \int_{\mathcal{D}_{\star}(\zeta)} g_{\mathcal{D}_{\star}(\zeta)}(w, \zeta) d\left(\beta \nu^{(k)}\right)(w) d \mu(\zeta)\right) \\
& =\frac{1+\alpha}{1-\alpha} \beta\left(\int_{\Omega} V_{\mu} d \nu+\int_{\Omega} \int_{\mathcal{D}_{\star}(\zeta)} g_{\mathcal{D}_{\star}(\zeta)}(w, \zeta) d \nu(w) d \mu(\zeta)\right) .
\end{aligned}
$$

By Proposition 6.2, for every $\mu \in J_{0}(\Omega) \cap \mathcal{M}_{\mathrm{ac}}^{+}(\Omega)$ we obtain

$$
\int_{\Omega} V_{\mu} d \lambda \leq \frac{1+\alpha}{1-\alpha} \beta \int_{\Omega} \mathcal{H}_{\mathcal{D}_{*}(\zeta)}^{p}(\zeta) d \mu(\zeta)-\frac{1+\alpha}{1-\alpha} \beta p(0) .
$$

This means that the assumption (1.7) of Theorem 1.2 is fulfilled with

$$
M(\zeta)=\frac{1+\alpha}{1-\alpha} \beta \mathcal{H}_{\mathcal{D}_{\star}(\zeta)}^{p}(\zeta), \quad C=-\frac{1+\alpha}{1-\alpha} \beta p(0)<+\infty
$$

(as was mentioned in Proposition 5.1, the function $M$ is locally integrable with respect to $m$ and $\mu$ ). Therefore, by Theorem [1.2, there is $h \in H(\Omega), h \neq \equiv 0$, such that

$$
\begin{aligned}
u(z)+\log |h(z)| \leq & \frac{1+\alpha}{1-\alpha} \beta \frac{1}{2 \pi} \int_{0}^{2 \pi}\left(\mathcal{H}_{\mathcal{D}_{\star}\left(z+t e^{i \theta}\right)}^{p}\right)^{(r)}\left(z+t e^{i \theta}\right) d \theta \\
& +\log (1+1 / t)+9 \log (1+|z|), \quad z \in \Omega, \quad 0<t<\operatorname{dist}(z, \partial \Omega) .
\end{aligned}
$$

Applying (5.8b) (see Proposition 5.3) to the right-hand side, we obtain (8.2).

Suppose $p \in S H(\Omega), p \not \equiv-\infty$, and $\mathcal{S}$ is a system of weights on $\Omega$. The following theorem is aimed at weighted classes of type $H_{\mathcal{P}}^{\uparrow}(\Omega)$ with $\mathcal{P}=p+\mathcal{S}$ in the role of the system of weights; in what follows, we use the notation $H_{p+\delta}(\Omega)$ for such classes. Clearly, these classes have a much more rigid structure than $A_{\mathcal{P}}^{\uparrow}(\Omega)$ or $H_{p}^{1}(\Omega)$ if the functions $s \in \mathcal{S}$ grow essentially more slowly than $p$ near $\partial \Omega$. In particular, if $\mathcal{S}$ consists of the functions of the form $C\left(\log \left(1+1 / d_{\Omega}(z)\right)+\log (1+|z|)\right)$, then $H_{p+\delta}(\Omega)$ is $H_{p+\log }(\Omega)$ (see the Introduction and (0.4)). 
Theorem 8.2 (Preparatory theorem). Under conditions (ii)-(iii) in $\S 7$, suppose that

$$
\begin{aligned}
& \limsup _{k \rightarrow \infty} \frac{\operatorname{diam} S_{k}}{\operatorname{dist}\left(S_{k}, \partial D_{k}\right)}<d<1, \\
& \lambda^{(k)}\left(S_{k}\right) \leq \nu^{(k)}\left(S_{k}\right)
\end{aligned}
$$

for all $k>k_{0}$. We introduce the measure $\sigma \in \mathcal{M}^{+}(\Omega)$ by

$$
\sigma \stackrel{\text { def }}{=} \frac{2}{1-d} \sum_{k=1}^{\infty} \frac{\operatorname{diam} S_{k}}{\operatorname{dist}\left(S_{k}, \partial D_{k}\right)} \nu^{(k)}
$$

Then for every function $s \in S H(\Omega)$ with Riesz measure $\sigma$ and for every function $r$ as in (7.3) and locally separated from zero, there exists a function $h \in H(\Omega), h \neq \equiv$, and a constant $B \geq 0$ such that, in the notation (4.3) and (5.4)-(5.6), for all $z \in \Omega$ we have

$$
\begin{aligned}
u(z)+\log |h(z)| \leq & \inf _{0<t<d_{\Omega}(z)}\left(\mathcal{H}_{\left(\mathcal{D}_{\star}[z, t)\right)^{r}}^{p}(z)+\mathcal{H}_{\left(\mathcal{D}_{\star}[z, t)\right)^{r}}^{s}(z)+\log (1+1 / t)\right) \\
& +B \log (1+|z|) .
\end{aligned}
$$

Proof. As before, we again assume without loss of generality that $r$ is a continuous function.

By (8.7ㅁ) , there exists $k_{1}>k_{0}$ such that $\operatorname{diam} S_{k} \leq d \operatorname{dist}\left(S_{k}, \partial D_{k}\right)$ for all $k \geq k_{1}$. In particular,

$$
\frac{\operatorname{diam} S_{k}}{\operatorname{dist}\left(S_{k}, \partial D_{k}\right)-\operatorname{diam} S_{k}} \leq \frac{\operatorname{diam} S_{k}}{(1-d) \operatorname{dist}\left(S_{k}, \partial D_{k}\right)}
$$

for $k \geq k_{1}$. We choose $N$ as in (7.6) and use the argument at the beginning of the proof of Theorem 7.1 to pass to the representation (7.7). By (7.13) and (7.14) with $A=1$, we easily eliminate the influence of $u_{0}$ and $p_{0}$. Therefore, there is no loss of generality in assuming that the family $\mathcal{D}$ is separated from zero, i.e., for some $\varepsilon>0$,

$$
D_{k} \cap D(\varepsilon)=\varnothing, \quad k=1,2, \ldots,
$$

the measures $\lambda^{(0)}$ and $\nu^{(0)}$ are absent in (7.1) (i.e., (6.1) is fulfilled), and, moreover, $u_{0}=p_{0}=0$ in (7.7) and the inequalities (8.78) and (8.10) are true for all $k \in \mathbb{N}$.

By (8.10) and Proposition 3.1, estimate (3.6) yields

$$
\sup _{z, w \in S_{k}} \tau_{D_{k}}(z, w) \leq 1+\frac{2}{1-d} \frac{\operatorname{diam} S_{k}}{\operatorname{dist}\left(S_{k}, \partial D_{k}\right)}=1+c_{k},
$$

which is also the definition of the numbers $c_{k}$. In particular, by (8.8) and (8.11) we have

$$
\sigma=\sum_{k=1}^{\infty} c_{k} \nu^{(k)}, \quad \operatorname{supp} \sigma \cap D(\varepsilon)=\varnothing .
$$

Under the adopted agreements, inequalities (8.78) coincide with (6.3). By Proposition 6.1 for every $\mu \in J_{0}(\Omega) \cap \mathcal{M}_{\mathrm{ac}}^{+}(\Omega)$ (with potential $V_{\mu}$ ), the inequalities mentioned above 
yield an estimate of type (6.4) (in view of (8.12)):

$$
\begin{aligned}
\int_{\Omega} V_{\mu} d \lambda \leq & \sum_{k=1}^{\infty}\left(1+c_{k}\right)\left(\int_{\Omega} V_{\mu} d \nu^{(k)}+\int_{\Omega} \int_{\mathcal{D}_{\star}(\zeta)} g_{\mathcal{D}_{\star}(\zeta)}(w, \zeta) d \nu^{(k)}(w) d \mu(\zeta)\right) \\
= & \sum_{k=1}^{\infty}\left(\int_{\Omega} V_{\mu} d \nu^{(k)}+\int_{\Omega} \int_{\mathcal{D}_{\star}(\zeta)} g_{\mathcal{D}_{\star}(\zeta)}(w, \zeta) d \nu^{(k)}(w) d \mu(\zeta)\right) \\
& +\sum_{k=1}^{\infty} c_{k}\left(\int_{\Omega} V_{\mu} d \nu^{(k)}+\int_{\Omega} \int_{\mathcal{D}_{\star}(\zeta)} g_{\mathcal{D}_{\star}(\zeta)}(w, \zeta) d \nu^{(k)}(w) d \mu(\zeta)\right) \\
= & I_{\nu}(\mu)+I_{\sigma}(\mu),
\end{aligned}
$$

where the quantities

$$
\begin{gathered}
I_{\nu}(\mu) \stackrel{\text { def }}{=} \int_{\Omega} V_{\mu} d \nu+\int_{\Omega} \int_{\mathcal{D}_{\star}(\zeta)} g_{\mathcal{D}_{\star}(\zeta)}(w, \zeta) d \nu(w) d \mu(\zeta), \\
I_{\sigma}(\mu) \stackrel{\text { def }}{=} \int_{\Omega} V_{\mu} d \sigma+\int_{\Omega} \int_{\mathcal{D}_{\star}(\zeta)} g_{\mathcal{D}_{\star}(\zeta)}(w, \zeta) d \sigma(w) d \mu(\zeta)
\end{gathered}
$$

are precisely the left-hand side in (6.6) (with $\nu$ replaced by $\sigma$ in the second case). The form of the quantities (8.15) and Proposition 6.2 show that, by (8.14), if $p$ and $s$ are functions whose Riesz measures are $\nu$ and $\sigma$ (respectively), then for every absolutely continuous Jensen measure $\mu$ we have, for $\Omega$,

$$
\begin{aligned}
\int_{\Omega} V_{\mu} d \lambda & \leq I_{\nu}(\mu)+I_{\sigma}(\mu) \\
& \leq\left(\int_{\Omega} \mathcal{H}_{\mathcal{D}_{\star}(\zeta)}^{p}(\zeta) d \mu(\zeta)-p(0)\right)+\left(\int_{\Omega} \mathcal{H}_{\mathcal{D}_{\star}(\zeta)}^{s}(\zeta) d \mu(\zeta)-s(0)\right) \\
& =\int_{\Omega}\left(\mathcal{H}_{\mathcal{D}_{\star}(\zeta)}^{p}(\zeta)+\mathcal{H}_{\mathcal{D}_{\star}(\zeta)}^{s}(\zeta)\right) d \mu(\zeta)+(-p(0)-s(0)),
\end{aligned}
$$

where $p(0) \neq-\infty$ by (8.11) and $s(0) \neq-\infty$ by (8.13). This means that the assumption (1.7) of Theorem 1.2 is fulfilled with $M(\zeta)=\mathcal{H}_{\mathcal{D}_{\star}(\zeta)}^{p}(\zeta)+\mathcal{H}_{\mathcal{D}_{\star}(\zeta)}^{s}(\zeta)$ (in Proposition 5.1 it was shown that this function is locally integrable with respect to $m$ and $\mu$ ). Therefore, by Theorem 1.2. there exists a function $h \in H(\Omega), h \not \equiv 0$, such that

$$
\begin{gathered}
u(z)+\log |h(z)| \\
\leq \frac{1}{2 \pi} \int_{0}^{2 \pi}\left(\left(\mathcal{H}_{\mathcal{D}_{\star}\left(z+t e^{i \theta}\right)}^{p}\right)^{(r)}+\left(\mathcal{H}_{\mathcal{D}_{\star}\left(z+t e^{i \theta}\right)}^{s}\right)^{(r)}\right)\left(z+t e^{i \theta}\right) d \theta \\
\quad+\log (1+1 / t)+9 \log (1+|z|) \\
\stackrel{(5.8 \mathrm{~b})}{\leq} \mathcal{H}_{\left(\mathcal{D}_{\star}[z, t)\right)^{r}}^{p}(z)+\mathcal{H}_{\left(\mathcal{D}_{\star}[z, t)\right)^{r}}^{s}(z)+\log (1+1 / t)+9 \log (1+|z|), \\
z \in \Omega, \quad 0<t<d_{\Omega}(z) .
\end{gathered}
$$

The next preparatory theorem is aimed at the case where the "gap" between weights determining a class of holomorphic functions is minimal (under the present approach; see the system of weights (0.4) and condition (L) in the Introduction).

Theorem 8.3 (Preparatory theorem). Under conditions (1i) -(iii) in $\S 7$, suppose that (8.7d) and (8.71) are fulfilled for all $k>k_{0}$, and let $\sigma$ be the measure from (8.8).

If $\sigma(\Omega)<+\infty$, then for every function $r$ locally separated from zero, satisfying (17.3), and having finite order if $\Omega$ is unbounded (i.e., $\lim \sup _{z \in \Omega, z \rightarrow \infty} \frac{\log r(z)}{\log |z|}<+\infty$ ), there 
exists $h \in H(\Omega), h \neq \equiv 0$, and a constant $B \geq 0$ such that, in the notation (4.3) and (5.4) - (5.6), we have

$$
u(z)+\log |h(z)| \leq \inf _{0<t<\max \left\{d_{\Omega}(z),|z|\right\}}\left(\mathcal{H}_{\left(\mathcal{D}_{\star}[z, t)\right)^{r}}^{p}(z)+\log \left(1+\frac{1}{t}\right)\right)+B \log (1+|z|) .
$$

If $\Omega$ is simply connected and its boundary contains more than one point, then the requirement for $\sigma_{0}$ to be finite can be replaced with the Blaschke condition (which is weaker):

$$
\int_{\Omega} g_{\Omega}(0, \zeta) d \sigma(\zeta)<+\infty, \quad g_{\Omega} \text { is the Green function of } \Omega .
$$

Proof. There is no loss of generality in assuming that (8.11) holds true and $\mu$ is supported off $D(\varepsilon)$, i.e., (8.13) is fulfilled.

Under the assumption that $\sigma$ is finite, consider the function

$$
s(z) \stackrel{\text { def }}{=} \int_{\mathbb{C}} \log \left|1-\frac{z}{w}\right| d \sigma(w)
$$

subharmonic in $\mathbb{C}$ and with Riesz measure $\sigma$. If $\sigma$ is finite, by (8.13) and [51, 6.7.2] we have

$$
s(z) \leq C \log ^{+} \frac{|z|}{\varepsilon}+C=l(z), \quad z \in \mathbb{C},
$$

where $C \geq 0$ is a constant and, as usual, $\log ^{+} x=\max \{0, \log x\}$. Since the function $l$ defined by (8.19) is harmonic off $D(\varepsilon)$, by (8.11) we have

$$
\mathcal{H}_{\left(\mathcal{D}_{\star}[z, t)\right)^{r}}^{s}(z) \leq \mathcal{H}_{\left(\mathcal{D}_{\star}[z, t)\right)^{r}}^{l}(z) \leq C^{\prime} \log (1+|z|+t+r(z))+C^{\prime}
$$

for some $D \Subset \Omega$ and all $z \in \Omega \backslash D$; increasing the constant $C^{\prime}>0$, we can extend (8.20) to all $z \in \Omega$.

But if $\sigma$ only satisfies the Blaschke condition (8.17) (and $\Omega$ has the properties mentioned before (8.17)), then for every subharmonic function $s_{\sigma}$ with Riesz measure $\sigma$, by [45. Theorem 4.5.4], there exists a harmonic majorant $H$ in $\Omega$. In other words, there is a holomorphic function $q$ with $\operatorname{Re} q=-H$; furthermore, the Riesz measure of the subharmonic function $s(z) \stackrel{\text { def }}{=} s_{\sigma}(z)+\operatorname{Re} q(z) \leq 0, z \in \Omega$, is $\sigma$, and (8.13) is true, that is, $s(0) \neq-\infty$. Thus, 8.20 is fulfilled for some function $s \in S H(\Omega)$ with Riesz measure $\sigma$ in any case. By Theorem 8.2 , for this choice of $s$ there is $h \in H(\Omega), h \not \equiv 0$, and a constant $B \geq 0$ such that

$$
\begin{aligned}
u(z)+\log |h(z)| \leq & \mathcal{H}_{\left(\mathcal{D}_{\star}[z, t)\right)^{r}}^{p}(z)+C^{\prime} \log (1+|z|+t+r(z))+C^{\prime} \\
& +\log \left(1+\frac{1}{t}\right)+B \log (1+|z|), \quad z \in \Omega, \quad 0<t<d_{\Omega}(z) .
\end{aligned}
$$

If necessary, we may increase $B$; using (7.3) , the restriction $t<\max \left\{d_{\Omega}(z),|z|\right\}$, and the assumption that $r$ is of finite order if $\Omega$ is unbounded, we arrive at (8.16) (possibly, after multiplication of $h$ by a small constant $a>0$ ).

Remark. It is possible to deduce (7.15) from (8.2) with $A=\frac{1+\alpha}{1-\alpha} \beta$, and from (8.16) with $A=1$, as in the remark at the end of $\S 7$.

If $\Omega$ is bounded, the summand $B \log (1+|z|) \leq B \log (1+\operatorname{diam} \Omega)=b$ can be dropped on the right in the final inequalities (17.4), (8.2), (8.9), and (8.16), because it can be incorporated in $h$ in the form of the factor $e^{-b}$.

Moreover, if $\Omega \neq \mathbb{C}$, then on the right in the same estimates, for every $z_{0} \in \mathbb{C} \backslash \Omega$, the summand $B \log (1+|z|)$ can be replaced with $([B]+1) \log \left(1+\frac{1}{\left|z-z_{0}\right|}\right)$, where $[B]$ is the integral part of $B$. For this, it suffices to multiply $h$ first by the function $\frac{1}{\left(z-z_{0}\right)^{[B]+1}}$ (holomorphic in $\Omega$ ) and then by a sufficiently small positive number. 


\section{REFERENCES}

[1] S. V. Shvedenko, Hardy classes and related spaces of analytic functions in the unit disc, polydisc and ball, Itogi Nauki i Tekhniki. Mat. Anal., vol. 23, VINITI, Moscow, 1985, pp. 3-124; English transl. in J. Soviet Math. 39 (1987), no. 6. MR0824267 (87h:30075)

[2] A. B. Aleksandrov, Function theory in the ball, Itogi Nauki i Tekhniki. Ser. Sovrem. Probl. Mat. Fundam. Napravleniya, vol. 8, VINITI, Moscow, 1985, pp. 115-190; English transl., Encyclopaedia Math. Sci., vol. 8, Springer-Verlag, Berlin, 1994, pp. 107-178. MR0850487 (88b:32002)

[3] H. Hedenmalm, Recent progress in the function theory of the Bergman space, Holomorphic Spaces (Berkeley, CA, 1995), Math. Sci. Res. Inst. Publ., No. 33, Cambridge Univ. Press, Cambridge, 1998, pp. 35-50. MR.1630644 (99e:46035)

[4] P. Colwell, Blaschke products. Bounded analytic functions, Univ. Michigan Press, Ann Arbor, 1985. MR0779463 (86f:30033)

[5] A. Djrbashian and F. A. Shamoian, Topics in the theory of $A_{\alpha}^{p}$ spaces, Teubner-Texte Math., Bd. 105, Teubner, Leipzig, 1988. MR1021691 (91k:46019)

[6] F. A. Shamoyan, A factorization theorem of M. M. Dzhrbashyan and the characteristic of zeros of functions analytic in the circle with a majorant of finite growth, Izv. Akad. Nauk Armyan. SSR Ser. Mat. 13 (1978), no. 5-6, 405-422; English transl. in Soviet J. Contemporary Math. Anal. 13 (1978), no. 5-6. MR0541789 (80i:30054)

[7] _ Zeros of functions analytic in the disk and growing near the boundary, Izv. Akad. Nauk Armyan. SSR Ser. Mat. 18 (1983), no. 1, 15-27; English transl., Soviet J. Contemporary Math. Anal. 18 (1983), no. 1, 13-25. MR0705983 (84g:30032)

[8] C. Horowitz, Zero sets and radial zero sets in function spaces, J. Anal. Math. 65 (1995), 145-159. MR1335372 (96h:30066)

[9] B. Korenblum, An extension of the Nevanlinna theory, Acta Math. 135 (1975), 187-219. MR0425124(54:13081)

[10] E. Beller, Factorization for non-Nevanlinna classes of analytic functions, Israel J. Math. 27 (1977), no. 3-4, 320-330. MR0442234 (56:620)

[11] E. Beller and C. Horowitz, Zero sets and random zero sets in certain function spaces, J. Anal. Math. 64 (1994), 203-217. MR1303512 (95j:30005)

[12] K. Seip, On a theorem of Korenblum, Ark. Mat. 32 (1994), 237-243. MR1277927 (95f:30054)

[13] _ On Korenblum's density condition for the zero sequences of $A^{-\alpha}$, J. Anal. Math. 67 (1995), 307-322. MR.1383499 (97c:30044)

[14] J. Bruna and X. Massaneda, Zero sets of holomorphic functions in the unit ball with slow growth, J. Anal. Math. 66 (1995), 217-252. MR1370351 (97f:32006)

[15] D. Luecking, Zero sequences for Bergman spaces, Complex Variables Theory Appl. 30 (1996), 345-362. MR1413164 (97g:30007)

[16] H. Hedenmalm, B. Korenblum, and K. Zhu, Theory of Bergman spaces, Grad. Texts in Math., vol. 199, Springer-Verlag, New York, 2000. MR1758653 (2001c:46043)

[17] O. Blasco, A. Kukuryka, and M. Nowak, Luecking's condition for zeros of analytic functions, Ann. Univ. Mariae Curie-Skłodowska Sect. A 58 (2004), 1-15. MR2199585 (2006k:30010)

[18] V. V. Napalkov, Spaces of analytic functions of given growth near the boundary, Izv. Akad. Nauk SSSR Ser. Mat. 51 (1987), no. 2, 287-305; English transl., Math. USSR-Izv. 30 (1988), no. 2, 263-281. MR0896998 (88g:46036)

[19] B. N. Khabibullin, Zero (sub)sets for spaces of holomorphic functions and (sub)harmonic minorants, Electronic Archive at LANL, 18 Dec. 2004, 42 pp., http://arxiv.org/abs/math.CV/0412359.

[20] _ Zero sequences of holomorphic functions, representation of meromorphic functions, and harmonic minorants, Mat. Sb. 198 (2007), no. 2, 121-160; English transl., Sb. Math. 198 (2007), no. 1-2, 261-298. MR2355445 (2008h:30054)

[21] Sets of uniqueness in spaces of entire functions of one variable, Izv. Akad. Nauk SSSR Ser. Mat. 55 (1991), no. 5, 1101-1123; English transl., Math. USSR-Izv. 39 (1992), no. 2, 1063-1084. MR:1149889(93e:30062)

[22] , A theorem on the least majorant and its applications. I. Entire and meromorphic functions, Izv. Ross. Akad. Nauk Ser. Mat. 57 (1993), no. 1, 129-146; English transl., Russian Acad. Sci. Izv. Math. 42 (1994), no. 1, 115-131. MR1220584 (94f:31003)

[23] _ Nonconstructive proofs of the Beurling-Malliavin theorem on the radius of completeness, and nonuniqueness theorems for entire functions, Izv. Ross. Akad. Nauk Ser. Mat. 58 (1994), no. 4, 125-148; English transl., Russian Acad. Sci. Izv. Math. 45 (1995), no. 1, 125-149. MR1307059 (96g:30005)

[24] P. Koosis, Leçons sur le théorème de Beurling et Malliavin, Univ. Montréal, Publ. CRM, Montreal, QC, 1996. MR.1430571 (99e:42023) 
[25] T. J. Ransford, Jensen measures, Approximation, Complex Analysis, and Potential Theory (Montreal, QC, 2000), NATO Sci. Ser. II Math. Phys. Chem., vol. 37, Kluwer Acad. Publ., Dordrecht, 2001, pp. 221-237. MR.1873590 (2002m:31019)

[26] B. N. Khabibullin, Dual approach to certain questions for the weighted spaces of holomorphic functions, Entire Functions in Modern Analysis (Tel-Aviv, 1997), Israel Math. Conf. Proc., vol. 15, Bar-Ilan Univ., Ramat Gan, 2001, pp. 207-219. MR1890538 (2003e:30009)

[27] _ Dual representation of superlinear functionals and its applications in function theory. II, Izv. Ross. Akad. Nauk Ser. Mat. 65 (2001), no. 5, 167-190; English transl., Izv. Math. 65 (2001), no. 5, 1017-1039. MR1874358 (2003b:46024)

[28] _ Completeness of systems of entire functions in spaces of holomorphic functions, Mat. Zametki 66 (1999), no. 4, 603-616; English transl., Math. Notes 66 (1999), no. 3-4, 495-506 (2000). MR 1747088 (2001d:32002)

[29] Estimates for the volume of null sets of holomorphic functions, Izv. Vyssh. Uchebn. Zaved. Mat. 1992, no. 3, 58-63. (Russian) MR1204818 (93k:32003)

[30] _ Uniqueness theorems for holomorphic functions, and balayage, Complex Analysis. Operator Theory. Mathematical Modelling, Vychisl. Nauchn. Tsentr. Ross. Akad. Nauk, Vladikavkaz, 2006, pp. 118-132. (Russian)

[31] L. Yu. Cherednikova and B. N. Khabibullin, Nonuniqueness sets for weighted algebras of functions holomorphic in the disk, Complex Analysis, Differential Equations and Related Problems. I. Complex Analysis, Trans. Internat. Conf., Ross. Akad. Nauk Ural. Nauchn. Tsentr. Inst. Mat. Vychisl. Tsentr, Ufa, 2000, pp. 195-200. (Russian)

[32] _ Nonuniqueness sequences for weighted algebras of functions holomorphic in the disk, Trudy Mat. Tsentr. Lobachevsk. 19 (2003), 221-223. (Russian)

[33] L. Yu. Cherednikova, Nonuniqueness sequences for weighted algebras of holomorphic functions in the unit disk, Mat. Zametki 77 (2005), no. 5, 775-787; English transl., Math. Notes 77 (2005), no. 5-6, 715-725. MR2178847(2006i:30074)

[34] L. Yu. Cherednikova and B. N. Khabibullin, Stability of nonuniqueness sequences for weighted algebras of functions holomorphic in the disk, Scientific Conf. on Scientific-Technical Programs of Education Ministry of the Russian Federation: Collected Works. Part I, Bashkir. Univ., Ufa, 2000, pp. 25-28. (Russian)

[35] B. N. Khabibullin and F. B. Khabibullin, Zero subsets for spaces of functions and the entropy of arcwise connectedness, Geometric Analysis and its Applications: Thesis of Internat. School-Conf., Volgograd. Univ., Volgograd, 2004, pp. 193-195.

[36] B. N. Khabibullin, Zero sets for weighted classes of holomorphic functions, Vestnik Bashkir. Univ. 2004, no. 2, 59-63. (Russian)

[37] L. Yu. Cherednikova, On "quenching" of the growth of subharmonic functions, Regional SchoolConf. for Students, Post Graduate Students, and Young Scientists in Mathematics and Physics. Vol. 1. Mathematics, Bashkir. Univ., Ufa, 2001, pp. 239-245. (Russian)

[38] W. K. Hayman and P. B. Kennedy, Subharmonic functions. Vol. 1, London Math. Soc. Monogr., No. 9, Acad. Press, London-New York, 1976. MR0460672 (57:665)

[39] T. W. Gamelin, Uniform algebras and Jensen measures, London Math. Soc. Lecture Note Ser., vol. 32, Cambridge Univ. Press, Cambridge-New York, 1978. MR0521440 (81a:46058)

[40] B. J. Cole and T. J. Ransford, Subharmonicity without upper semicontinuity, J. Funct. Anal. 147 (1997), 420-442. MR1454488 (98h:31005)

[41] _ Jensen measures and harmonic measures, J. Reine Angew. Math. 541 (2001), 29-53. MR.1876284 (2003c:31002)

[42] B. N. Khabibullin, Criteria for (sub-)harmonicity and the continuation of (sub-)harmonic functions, Sibirsk. Mat. Zh. 44 (2003), no. 4, 905-925; English transl., Siberian Math. J. 44 (2003), no. 4, 713-728. MR2010135 (2004g:31004)

[43] O. V. Epifanov, Solvability of the inhomogeneous Cauchy-Riemann equation in classes of functions that are bounded with weight and a system of weights, Mat. Zametki 51 (1992), no. 1, 83-92; English transl., Math. Notes 51 (1992), no. 1-2, 54-60. MR.1165283 (93h:30060)

[44] L. Hörmander, Notions of convexity, Progr. Math., vol. 127, Birkhäuser Boston, Inc., Boston, MA, 1994. MR1301332 (95k:00002)

[45] T. J. Ransford, Potential theory in the complex plane, London Math. Soc. Student Texts, vol. 28, Cambridge Univ. Press, Cambridge, 1995. MR1334766 (96e:31001)

[46] J. B. Garnett, Bounded analytic functions, Pure Appl. Math., vol. 96, Acad. Press, Inc., New York-London, 1981. MR0628971 (83g:30037) 
[47] L. Yu. Cherednikova, Elementary estimates in terms of Harnack distances, Regional School-Conf. for Students, Post Graduate Students, and Young Scientists in Mathematics and Physics. Vol. II. Mathematics, Bashkir. Univ., Ufa, 2002, pp. 87-90. (Russian)

[48] M. Brelot, Eléments de la théorie classique du potentiel, Centre Doc. Univ., Paris, 1959. MR0106366 (21:5099)

[49] _ On topologies and boundaries in potential theory, Lecture Notes in Math., vol. 175, Springer-Verlag, Berlin-New York, 1971. MR0281940 (43:7654)

[50] A. V. Arkhangel'skiǐ and V. I. Ponomarev, Fundamentals of general topology. Problems and exercises, "Nauka", Moscow, 1974; English transl., D. Reidel Publ. Co., Dordrecht, 1984. MR0445439 (56:3781) MR:0785749 (87i:54001)

[51] W. K. Hayman, Subharmonic functions. Vol. 2, London Math. Soc. Monogr., vol. 20, Acad. Press, London, 1989. MR1049148(91f:31001)

Bashkir State University, Institute of Mathematics with Computer Center, Urals Scientific Center, Russian Academy of Sciences, Ufa, Bashkortostan, Russia

E-mail address: khabib-bulat@mail.ru

Bashkir State University, Institute of Mathematics with Computer Center, Urals Scientific Center, Russian Academy of Sciences, Ufa, Bashkortostan, Russia

Bashkir State University, Institute of Mathematics with Computer Center, Urals Scientific Center, Russian Academy of Sciences, Ufa, Bashkortostan, Russia

Received 6/NOV/2006

Translated by S. KISLYAKOV 